\title{
3D edge transport analysis of ITER start-up configuration for limiter power load assessment
}

M Kobayashi ${ }^{1}$, Y Feng ${ }^{2}$, A Loarte ${ }^{3}$, G Federici ${ }^{4}$, G Strohmayer ${ }^{4}$, M. Shimada $^{5}$, F Sardei ${ }^{2}$, D Reiter ${ }^{6}$, and M. Sugihara ${ }^{5}$

1 National Institute for Fusion Science,322-6 Oroshi, Toki, 509-5292, Japan

2 Max-Planck-Institut für Plasmaphysik,EURATOM Association, 17491 Greifswald, Germany

3 EFDA-CSU Garching, Boltzmannstr. 2, D-85748 Garching, Germany

4 ITER JWS Garching Co-center, Boltzmannstraße 2, 85748 Garching, Germany

${ }^{5}$ ITER JWS Naka Co-centre, 801-1 Mukouyama, Naka-machi, Naka-gun, Ibaraki-ken, Japan

${ }^{6}$ Institute of Plasma Physics, Forschungszentrum Jülich GmbH, 52425 Jülich, Germany

E-mail : kobayashi.masahiro@LHD.nifs.ac.jp

\begin{abstract}
The edge transport properties of the toroidally discrete limiter configuration in the ITER start-up phase has been analyzed, using the 3D edge transport code, EMC3EIRENE. Because of the finite magnetic shear in the edge, the interaction of the limiters with flux surfaces of different q-values introduces a complex 3D pattern in the connection length $\left(\mathrm{L}_{\mathrm{C}}\right)$ profiles, where long and short flux tubes co-exist in the scrape-off layer. The severity of problems associated with very long flux tubes in the edge, which could bring a large amount of energy (proportional to square root of $\mathrm{L}_{\mathrm{C}}$ ), and cause a hot spot on the limiter, was mitigated and no significant localized power load was found. This can be justified as follows: (i) For long flux tubes, the perpendicular energy transport time becomes shorter than the parallel energy transport time, resulting in no net energy input to the flux tube. (ii)Perpendicular transport was found to be very effective to smear out the difference in parallel energy flux conducted by the various flux tubes, if they interact within a perpendicular transport scale, $\sim$ a few $\mathrm{cm}$, which is usually the case in high plasma current ITER start-up configuration. These two effects significantly reduce the dependence of energy deposition on $\mathrm{L}_{\mathrm{C}}$. At the high plasma current (e.g. 6.5 MA), the peak power load is found to be close to the engineering limit, especially for lowest perpendicular transport coefficients and the highest input power. Comparing the results of the 3D modelling with a radial exponential decay model, it was found that by neglecting the 3D geometrical effects, the simple model overestimates peak power load by $\sim 30 \%$ for corresponding input power and radial decay of energy flux.
\end{abstract}

PACS : 52.55.Fa, 52.55.Rk, 52.25.Fi, 52.65.Pp, 52.65.Kj, 52.65.-y 


\section{Introduction}

Two beryllium limiter modules, toroidally localized at the low field side (LFS), are foreseen to be used to sustain the plasma start-up phase in the current ITER design, as shown in Fig. 1. During this phase that lasts for about $20 \sim 30$ seconds before the $\mathrm{X}$ point divertor configuration is fully established, the role of the limiters is to define plasma boundary in order to protect the first wall from a direct exposure to plasma heat flux. Each limiter has a surface area of $\sim 3 \mathrm{~m}^{2}$, which intercepts a power flow to the limiter scrape-off layer (SOL) of several MW. The limiter shape has to be optimized to spread the energy flux effectively over the surface, in order to keep the peak power load below the engineering limit, $8 \mathrm{MW} / \mathrm{m}^{2}[1,2]$. The following terms, among many other edge transport processes, are to be considered for this purpose.
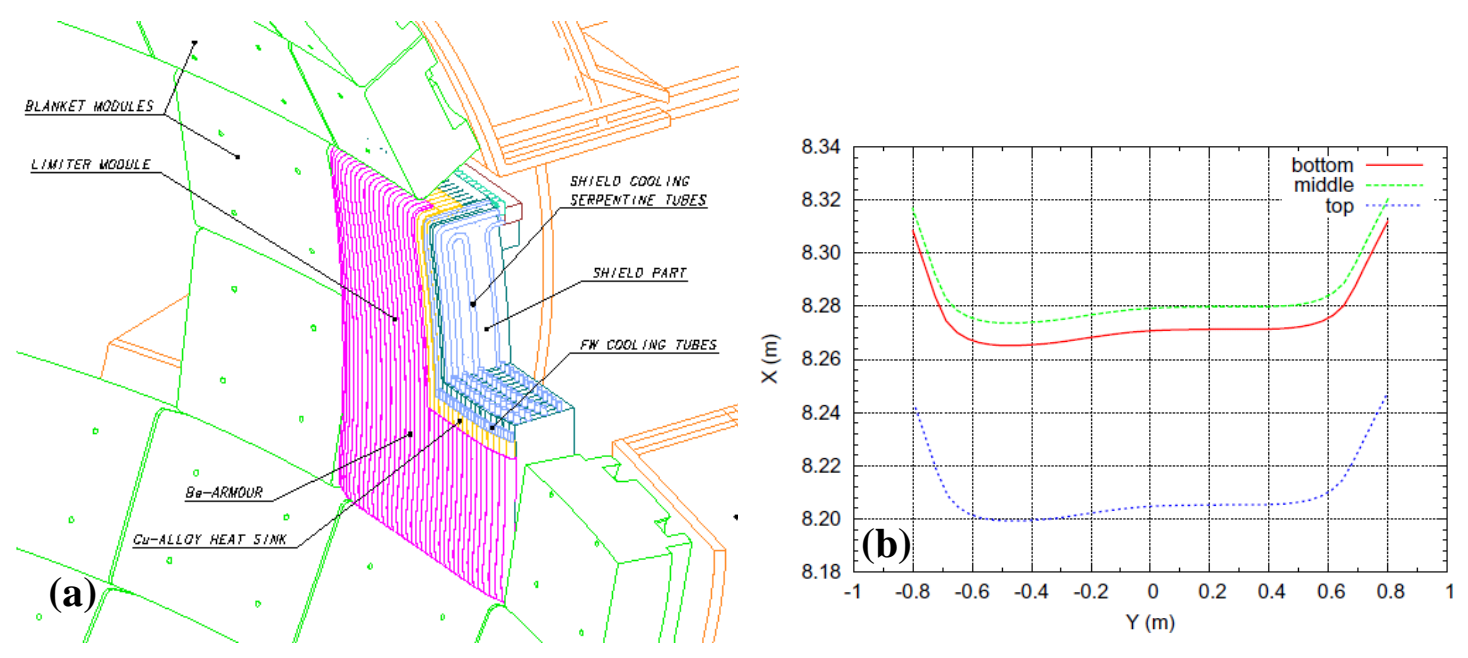

Fig. 1 (a) The start-up limiter at the LFS indicated by red color. The toroidal extent is $\Delta \phi \sim 12$ deg. $(1.6 \mathrm{~m}), \phi$ toroidal angle, and poloidal extent is $\sim 2.1 \mathrm{~m}$. (b) horizontal cut of the limiter at different height, top, middle and bottom. The Y axis is a horizontal line tangential to the flux surface at $\phi=0$ deg., i.e. almost corresponds to the toroidal direction, but with opposite sign $(\mathrm{Y}>0$ for $\phi<0)$. The $\mathrm{X}$ axis is horizontal one orthogonal to $\mathrm{Y}$.

I. Since the limiters are localized in toroidal direction (the toroidal extent is $\Delta \phi \sim 12$ deg. (1.6 m) and poloidal extent is $\sim 2.1 \mathrm{~m}$ ), they can not cut the flux tubes perfectly, especially at rational $\mathrm{q}$ surfaces. This gives rise to flux tubes with very long connection length $\left(\mathrm{L}_{\mathrm{C}}\right)$. From a simple consideration between parallel and perpendicular power flow in a flux tube, one could obtain a relation,

$$
\lambda_{p} \propto \sqrt{\left(2.5 D_{\perp}+\chi_{\perp}\right) L_{C}},
$$

where $\lambda_{\mathrm{p}}, D_{\perp}$ and $\chi_{\perp}$ are the power fall-off length, perpendicular particle and heat diffusivities, respectively. One sees that the longer the $\mathrm{L}_{C}$ is, the larger the SOL width $\left(\lambda_{\mathrm{p}}\right)$ becomes. The larger $\lambda_{\mathrm{p}}$ will help spread the power load on the limiter surface, but at the same time, this may result in a leading edge problem or power deposition on the first wall.

II. As the $\mathrm{L}_{\mathrm{C}}$ becomes longer, one would expect that the flux tubes are fed with more energy while they travel long around the main plasma. A simple estimate leads to

$$
\Gamma_{/ /} \propto \sqrt{L_{C}},
$$


where $\Gamma_{/ /}$is the parallel power flux in the flux tubes. When these flux tubes hit the limiter, they may cause hot spots.

In reality, because of cross field transport there is an exchange of energy between flux tubes with different $\mathrm{L}_{\mathrm{C}}$ 's, which could reduce the severity of problems associated with very long flux tubes (eq. (1-2) ). As shown later (section 3), the $\mathrm{L}_{\mathrm{C}}$ profile in the toroidally discrete limiter configuration exhibits a rather complex three dimensional structure, and the transport process seems to be complex as well through the various associated geometrical effects.

III. As shown in eq.(1-1), the SOL width depends on the perpendicular transport coefficients, which are yet unknown for ITER. To confirm the engineering margins of the present design, these coefficients need to be scanned within ranges, based on an experimental database.

In the previous assessment [3], the limiter was designed based on a simplified model, which assumes an exponential radial decay of $\Gamma_{/ /}$resulting from a value of $\lambda_{\mathrm{p}}$ assumed a priori. It ensured that the peak power load was marginally less than the critical design value (at which melting or sublimation will occur) for a nominal range of $\lambda_{\mathrm{p}}$ and misalignments of the limiters. Because of the complexity of geometry as well as of the transport processes mentioned above, however, it was felt to readdress this issue taking into account all the terms in more realistic way.

In this paper, the edge transport properties in the limiter configuration are analyzed by using the 3D edge transport code, EMC3-EIRENE [4,5], in order to treat the transport processes mentioned above in a self-consistent manner, and to improve confidence of the previous estimation for determining $\lambda_{\mathrm{p}}$ and the peak power loads.

\section{Description of EMC3-EIRENE}

EMC3 [4] solves a set of standard Braginskii equations of mass, momentum and energy (electron \& ion),

$$
\begin{aligned}
& \nabla_{/ /} \cdot\left(n V_{/ /}\right)+\nabla_{\perp} \cdot\left(-D_{\perp} \nabla_{\perp} n\right)=S_{p}, \quad(2-1) \\
& \nabla_{/ /} \cdot\left(m_{i} n V_{/ /} V_{/ /}-\eta_{/ /} \nabla_{/ /} V_{/ /}\right)+\nabla_{\perp} \cdot\left(-m_{i} V_{/ /} D_{\perp} \nabla_{\perp} n-\eta_{\perp} \nabla_{\perp} V_{/ /}\right)=-\nabla_{/ /} n\left(T_{e}+T_{i}\right)+S_{m}, \quad(2-2) \\
& \nabla_{/ /} \cdot\left(-\kappa_{/ /}^{e} \nabla_{/ /} T_{e}+\frac{5}{2} n T_{e} V_{/ /}\right)+\nabla_{\perp} \cdot\left(-\chi_{\perp}^{e} n \nabla_{\perp} T_{e}-\frac{5}{2} T_{e} D_{\perp} \nabla_{\perp} n\right) \\
& =-k\left(T_{e}-T_{i}\right)+S_{e e} \quad(\text { electron }), \quad(2-3) \\
& \left.\nabla_{/ /} \cdot\left(-\kappa_{/ /}^{i} \nabla_{/ /} T_{i}+\frac{5}{2} n T_{i} V_{/ /}\right)+\nabla_{\perp} \cdot\left(-\chi_{\perp}^{i} n \nabla_{\perp} T_{i}-\frac{5}{2} T_{i} D_{\perp} \nabla_{\perp} n\right)=k\left(T_{e}-T_{i}\right)+S_{e i} \quad \text { (ion), (2- }\right)
\end{aligned}
$$

in a realistic 3D geometry of magnetic field and plasma facing components. In the equations, $V_{/ /}$, $\kappa^{\rho, i} / /$ and $k$ are the parallel plasma velocity, classical parallel heat conductivity of electron/ion and the equilibration coefficient, respectively. The perpendicular transport coefficients for particles 
and energy, $D_{\perp}$ and $\chi_{\perp}$, are assumed to be anomalous and given as an input parameter. The perpendicular viscosity coefficient $\eta_{\perp}$ is set as $\mathrm{m}_{\mathrm{i}} \mathrm{n} D_{\perp} . S_{p}, S_{m}, S_{e e}$ and $S_{e i}$ are the ionization source, momentum source/loss, energy source/loss via atomic/molecular processes e.g. charge exchange (CX) and ionization, which are calculated by EIRENE [5]. At the limiter surface, the Bohm condition is assumed as a particle/energy loss flux. The particles are reflected as recycling neutrals with a recycling coefficient of unity.

The present analysis was conducted for the limiter configuration of the start-up scenario 2 for three snapshots of the plasma current $\left(\mathrm{I}_{\mathrm{p}}\right)$ of 2.5, 4.5 and 6.5 MA. (For this scenario, a divertor configuration is achieved at 6.5 MA and also possible at 4.5 MA. [6]) The computational domain for Ip $=6.5 \mathrm{MA}$ is shown in Fig. 2, where the domain for plasma is indicated by blue lines and the limiter shape is shown with a red line. The domain for neutrals is extended up to the first wall. The power flow to the SOL, $\mathrm{P}_{\mathrm{SOL}}$, and the up-stream density (density at the inner boundary, $\mathrm{n}_{\text {up }}$ ) are given by the core transport analysis described in Ref. [7]. $\mathrm{P}_{\mathrm{SOL}}$ is assumed for the time being to be symmetrically divided to electrons and ions. At the inner boundary, density is fixed to $\mathrm{n}_{\mathrm{up}}$ while for energy transport, energy flux is given as a boundary condition. At the outer boundary, certain decay lengths, a few $\mathrm{cm}$, are defined for loss flux boundary condition. Drift, e.g. ExB and grad B drifts, is not included in the model. The recycling neutrals are generated on the limiter surface weighted with the particle deposition pattern and traced for experiencing the atomic/molecular processes determined by EIRENE. Several benchmark and control tests of EMC3 with a realistic 3D geometry of the ITER configuration were done to ensure a feasibility of the code and accuracy for transport analysis.

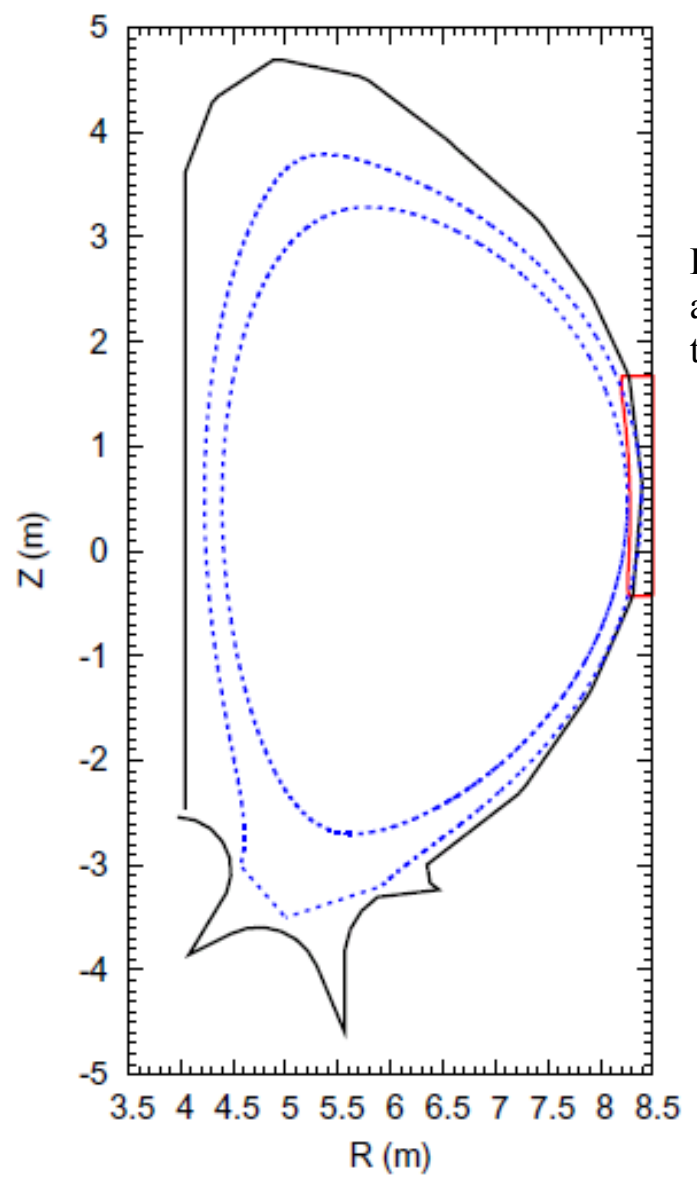

Fig. 2 Computational domain of the edge transport analysis for Ip $=6.5 \mathrm{MA}$ depicted with blue lines, together with the limiter (red) and the first wall (black). 


\section{Magnetic field structure}

In the present analysis, the two limiters are assumed to be located in toroidal symmetric places at $\phi$ (toroidal angle) $=0$ and $180 \mathrm{deg}$. The connection length profiles for the different Ip's are shown in Fig. 3 in the poloidal plane at $\phi=0$ deg. (centre of the limiter), where the $L_{C}$ is a distance measured from limiter to limiter. The poloidal and radial coordinates are measured with the toroidal magnetic flux. $r_{\text {mag }}=0$ and 1 correspond to the inner and the outer computational domain (Fig. 2), while $\theta_{\mathrm{mag}}=0$ and 1 to $\theta=0$ and $2 \pi$ at the HFS, respectively. The limiter is located at $\theta_{\mathrm{mag}} \sim 0.5$ (LFS) (shown in Fig. 3 with a white background, protruding up to $\mathrm{r}_{\mathrm{mag}} \sim 0.2$ ). Also shown in Fig. 4 are the q profiles for different values of plasma current. Around the qrational surfaces because of the periodic transit of field lines there will be some flux tubes which never strike the limiter. For example, around $\mathrm{r}_{\mathrm{mag}}=0.3$ for $2.5 \mathrm{MA}$ with $\mathrm{q}=5$, there appears 9 regions with $\mathrm{L}_{\mathrm{C}} \sim 2 \pi \mathrm{Rq}(\sim 200 \mathrm{~m}$, blue coloured $), 5$ coming from opposite limiter, together with very long $\mathrm{L}_{\mathrm{C}}$ (more than $10000 \mathrm{~m}$, yellow coloured).

By moving outward radially, other resonances are encountered, e.g. at $\mathrm{r}_{\mathrm{mag}} \sim 0.6$ with $\mathrm{q}=5.5$, (i.e. $\mathrm{m} / \mathrm{n}=11 / 2$ ) producing 10 short $\mathrm{L}_{\mathrm{C}}$ regions. The similar resonance appears at $\mathrm{r}_{\text {mag }} \sim 0.2$ for 4.5MA with $\mathrm{q}=5$ and $\mathrm{r}_{\mathrm{mag}} \sim 0.3$ for $6.5 \mathrm{MA}$ with $\mathrm{q}=6$, respectively, as shown in Fig. 3 and 4 . The short $\mathrm{L}_{\mathrm{C}}$ region becomes narrow in poloidal direction going towards high $\mathrm{I}_{\mathrm{p}}$, where the LCFS becomes larger, because of the associated decrease of the relative poloidal extent of limiter to the plasma size. The ensuing decrease in radial direction is due to the increase of the shear (q') as shown in Fig. 4. Especially at $\mathrm{Ip}=6.5 \mathrm{MA}$, due to the loss of $\mathrm{q}=5$ surface cut by limiter, the $\mathrm{L}_{\mathrm{C}}$ becomes longer on average. One can observe that because of these resonant effects, the magnetic field structure exhibits a rather complex 3D pattern, in which very long flux tubes are intermixed with short ones and mutual interaction is expected via cross-field transport (see next section). 

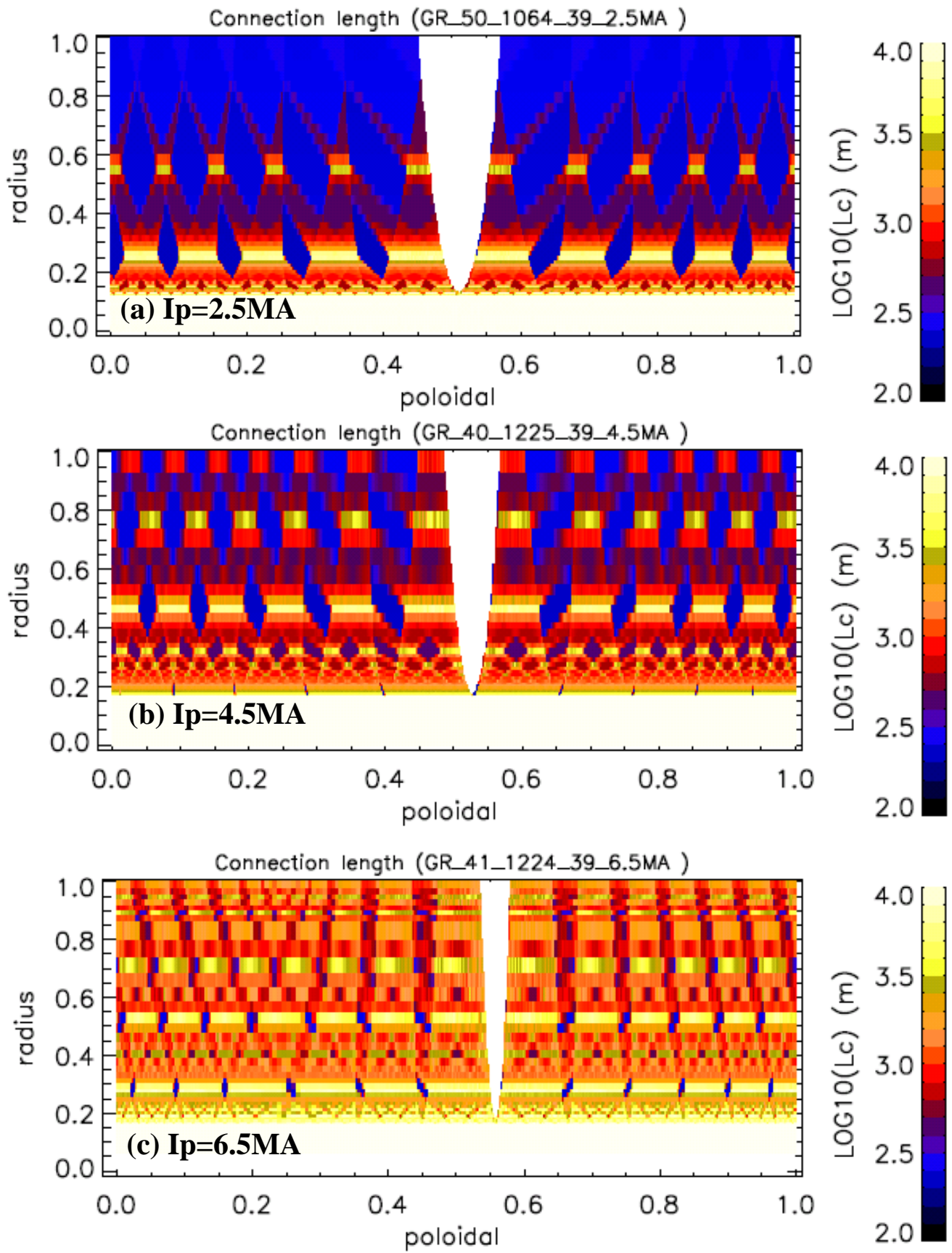

Fig. 3 Connection length profiles for different Ip's, (a) 2.5MA, (b) 4.5MA, (c) 6.5MA, in poloidal plane at $\phi=0$ deg. (centre of the limiter) with $\mathrm{L}_{\mathrm{C}}$ in logarithmic scale. $\mathrm{L}_{\mathrm{C}}$ is a distance measured from limiter to limiter. The radial and poloidal coordinates are measured with the toroidal magnetic flux. $r_{\text {mag }}=0$ and 1 correspond to inner and outer computational domain (Fig. 2), while $\theta_{\text {mag }}=0$ and 1 to $\theta=0$ and $2 \pi$ at HFS, respectively. The limiter is depicted with white background at $\theta_{\text {mag }} \sim 0.5$ (LFS), protruding up to $\mathrm{r}_{\mathrm{mag}} \sim$ 0.2 . 


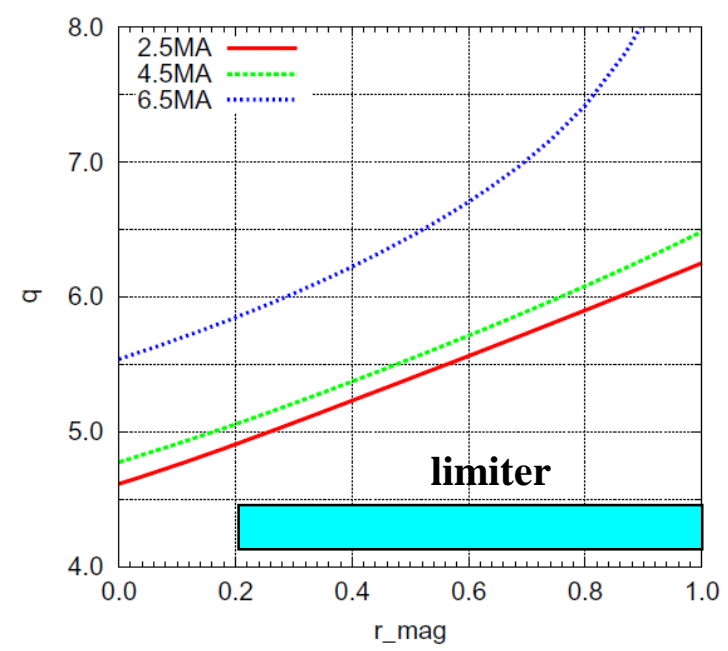

Fig.4 q profiles for different Ip's, 2.5, 4.5 and 6.5 MA. The limiter protrudes up to $\mathrm{r}_{\mathrm{mag}} \sim 0.2$.

\section{Transport properties in discrete limiter configuration}

\subsection{Results of EMC3-EIRENE}

The plasma parameter profiles at $\phi=0 \mathrm{deg}$. (centre of limiter) in poloidal plane obtained by EMC3-EIRENE for the case of Ip $=6.5 \mathrm{MA}, \mathrm{P}_{\mathrm{SOL}}=6 \mathrm{MW}$, up stream density, $\mathrm{n}_{\text {up }}=0.54 \times 10^{19}$ $\mathrm{m}^{-3}, D_{\perp}=0.4 \mathrm{~m}^{2} / \mathrm{s}, \chi_{\perp}=1.6 \mathrm{~m}^{2} / \mathrm{s}$ are plotted in Fig.5. The evaluation of power flowing to the limiter during start-up phase is described elsewhere [7]. For the purpose of this study a total power to the limiter modules of $4 \mathrm{MW}$ is obtained, but in order to cover the remaining uncertainties values up to $6 \mathrm{MW}$ were considered (also see section 5, Parameter scan study). There appears a strong poloidal modulation because of the shadow of the limiter, where the connection length becomes short. The modulations are better seen in the ion temperature rather than electron temperature because of the lower parallel heat conductivity the former. In the shadow regions, the flow towards the limiter is accelerated by the Bohm condition at the surface. The yellow color in the Mach number indicates the flow in positive direction of $\phi$ (counter clockwise viewed from top of torus) and the dark blue does in negative one. The flows are induced via viscosity also in the neighbouring flux tubes which are not connected to the limiter. Coming from both sides (positive and negative $\phi$ ), they meet in front of the limiter and stagnate there. This gives rise to a density increase in consequence of the pressure rise to balance the friction forces from the SOL flows due to the cross-field shear-viscous transport. It is also found that the $70 \%$ of the recycling neutrals are ionized in the SOL and the neutrals are well localized near the limiter.

The transport coefficient, $D_{\perp}$, was scaled with respect to the plasma current from estimated values obtained in JET limiter discharges [8,9]. For the present computations, $\chi_{\perp}$ was set such that the ratio of decay length of $\mathrm{T}_{\mathrm{e}}$ and $\mathrm{n}, \lambda_{T e} / \lambda_{n}$, becomes $1 \sim 1.5$, based on the measurement in JET. It was also found that $\chi_{\perp}=2 \sim 4 D_{\perp}$. Radial profiles of $\mathrm{T}_{\mathrm{e}}, \mathrm{T}_{\mathrm{i}}$ and $\mathrm{n}$ are plotted in Fig. 6 (a), where the radial coordinate is referred to the outer mid-plane. The $T_{e}, T_{i}$ and $n$ profiles are averaged along the flux surfaces over the poloidal and toroidal directions. Although the profiles are not simple exponential function, the ratio, $\lambda_{T e} / \lambda_{n}$, follows roughly the experimental value, $\sim$ 1.6 . 


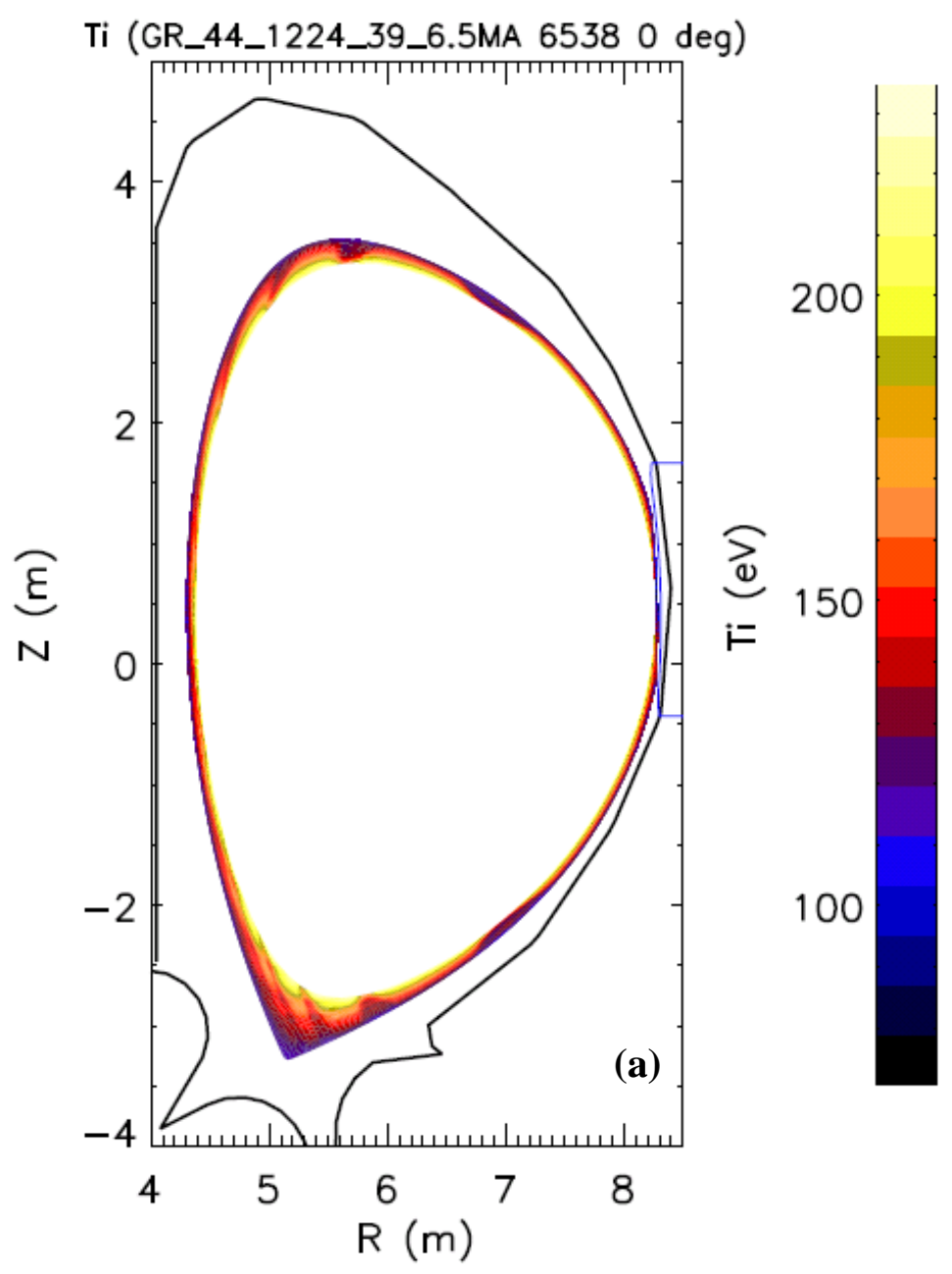




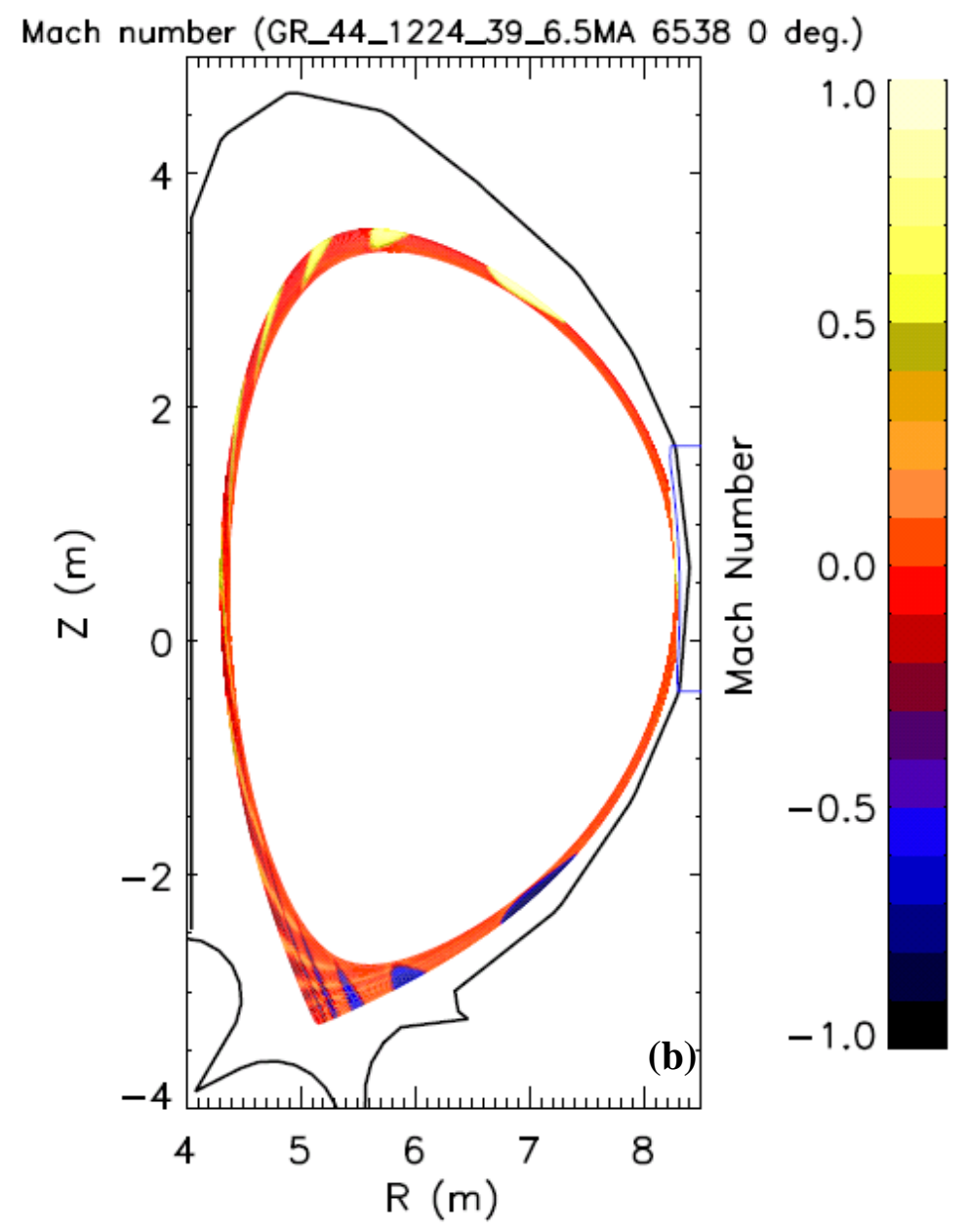

Fig. 5 Plasma parameter profiles in poloidal plane at $\phi=0$ deg. obtained by EMC3-EIRENE, (a) $\mathrm{Ti}$, (b) Mach number, for the case of Ip $=6.5 \mathrm{MA}, \mathrm{P}_{\mathrm{SOL}}=6 \mathrm{MW}$, up stream density, $\mathrm{n}_{\text {up }}=0.54 \mathrm{x}$ $10^{19} \mathrm{~m}^{-3}, D_{\perp}=0.4 \mathrm{~m}^{2} / \mathrm{s}, \chi_{\perp}=1.6 \mathrm{~m}^{2} / \mathrm{s}$. In the Mach number, yellow color indicates a flow in positive $\phi$ direction (counter clockwise viewed from top of torus) and dark blue for negative direction.
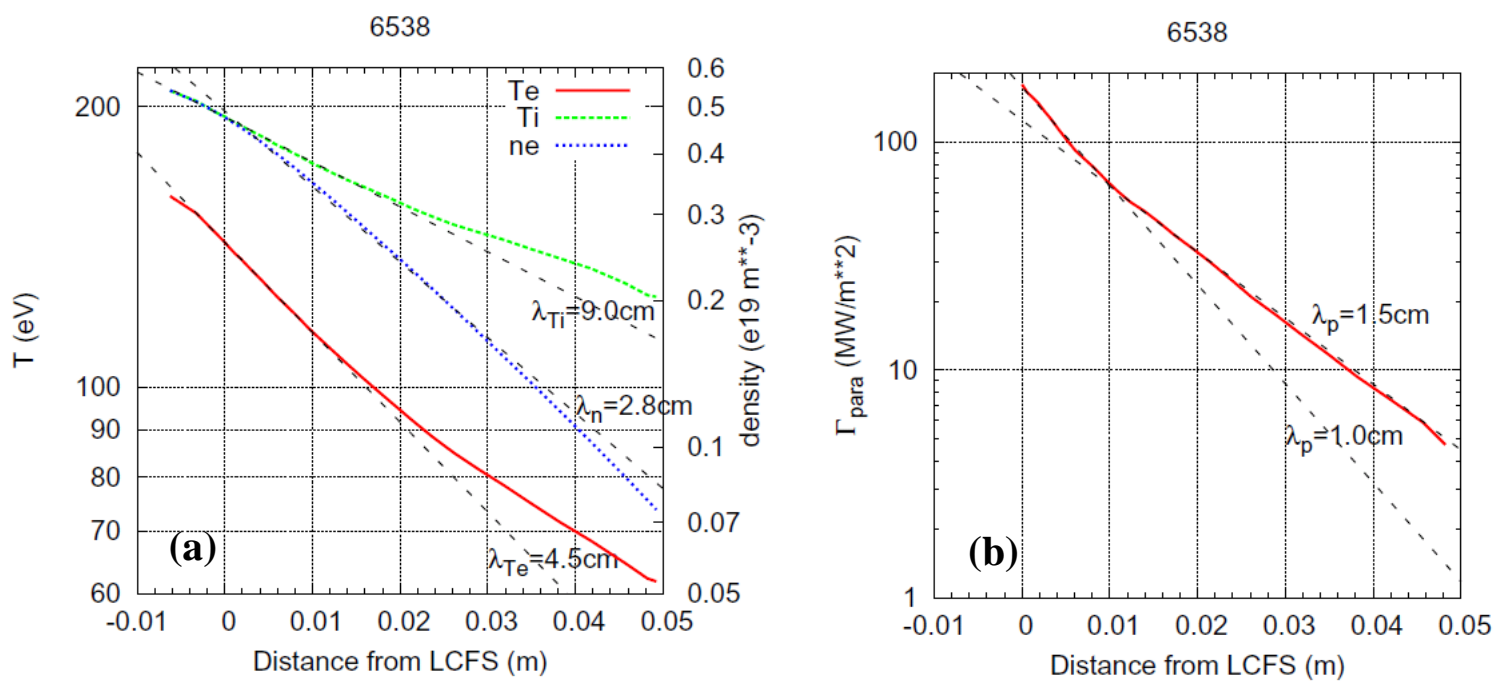
Fig. 6 Radial profiles of (a) $T_{e}, T_{i}$ and $n$ averaged along flux surfaces in poloidal and toroidal directions, and (b) the parallel energy flux to the limiter averaged in poloidal direction. The radial coordinate is referred to the outer midplane.

The parallel energy flux to the limiter, $\Gamma_{/ /}$, which is averaged in poloidal direction, is plotted in Fig. 6 (b). $\Gamma_{/ /}$changes the decay length around $\mathrm{r}=0.01 \mathrm{~m}$ from $\lambda_{\mathrm{p}}=1 \mathrm{~cm}$ to $1.5 \mathrm{~cm}$, which corresponds to the change of $T_{e}$ and $T_{i}$ profiles in Fig. 6 (a). The profiles imply that the power deposition is not simply approximated with an exponential decay function, but rather one needs a careful treatment of the 3D geometry of magnetic field structure. The effect of the 3D geometry on the transport process is discussed in the next section.

\subsection{Energy transport process}

The energy deposition pattern on the limiter is shown in Fig. 7 (a) together with (b) $\mathrm{L}_{\mathrm{C}}$ profile on the limiter surface. The axis $\mathrm{Y}$ is a horizontal line tangential to the flux surface at $\phi=0$ deg., i.e. corresponds almost to the toroidal direction. The deposition pattern is divided into two regions around $\mathrm{Y}=0$, where the energy deposition becomes small with decreasing incidence angle of flux tubes to the limiter surface. Since the limiter protrudes more at the $\mathrm{Y}<0 \mathrm{~m}$ as shown in Fig. 1 (b), the deposition on this side is larger than the side of $Y>0$ m. Compared with Fig. 7 (b), there is no significant correlation with the $\mathrm{L}_{\mathrm{C}}$ profiles identified, against the prediction of eq. (1-2).

The $T_{e}$ profile is plotted in $r_{\text {mag }}$ and $\theta_{\text {mag }}$ coordinates in Fig. 8. It is found that there is no clear correlation between $T_{e}$ and $L_{C}$ pattern (see Fig. 3 (c) or Fig. 12) except for the main resonance, i.e. except for the shadow of the limiter produced by $q=6$ flux surface. This indicates that the plasma smears out the difference of $\mathrm{L}_{\mathrm{C}}$ 's, which is finer than the main resonance, via cross-field transport. The dependency of eq.(1-2) is then reduced. In the following, the transport process is discussed. 

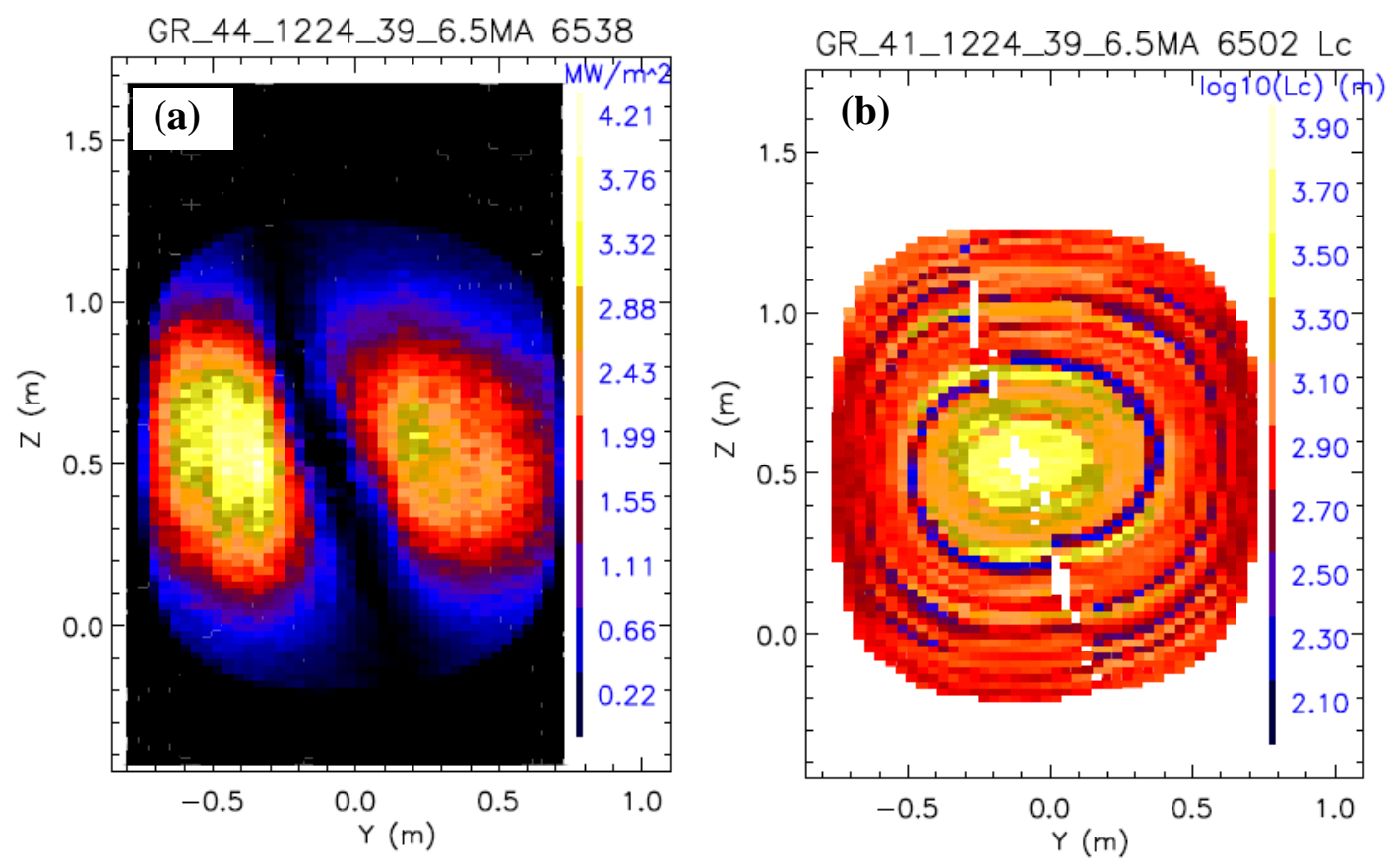

Fig. 7 (a) Energy deposition, (b) $\mathrm{L}_{\mathrm{C}}$ profile on the limiter surface. The $\mathrm{Y}$ axis is a horizontal line tangential to the flux surface at $\phi=0$ deg., i.e. almost corresponds to the toroidal direction, but with opposite sign $(\mathrm{Y}>0$ for $\phi<0)$.

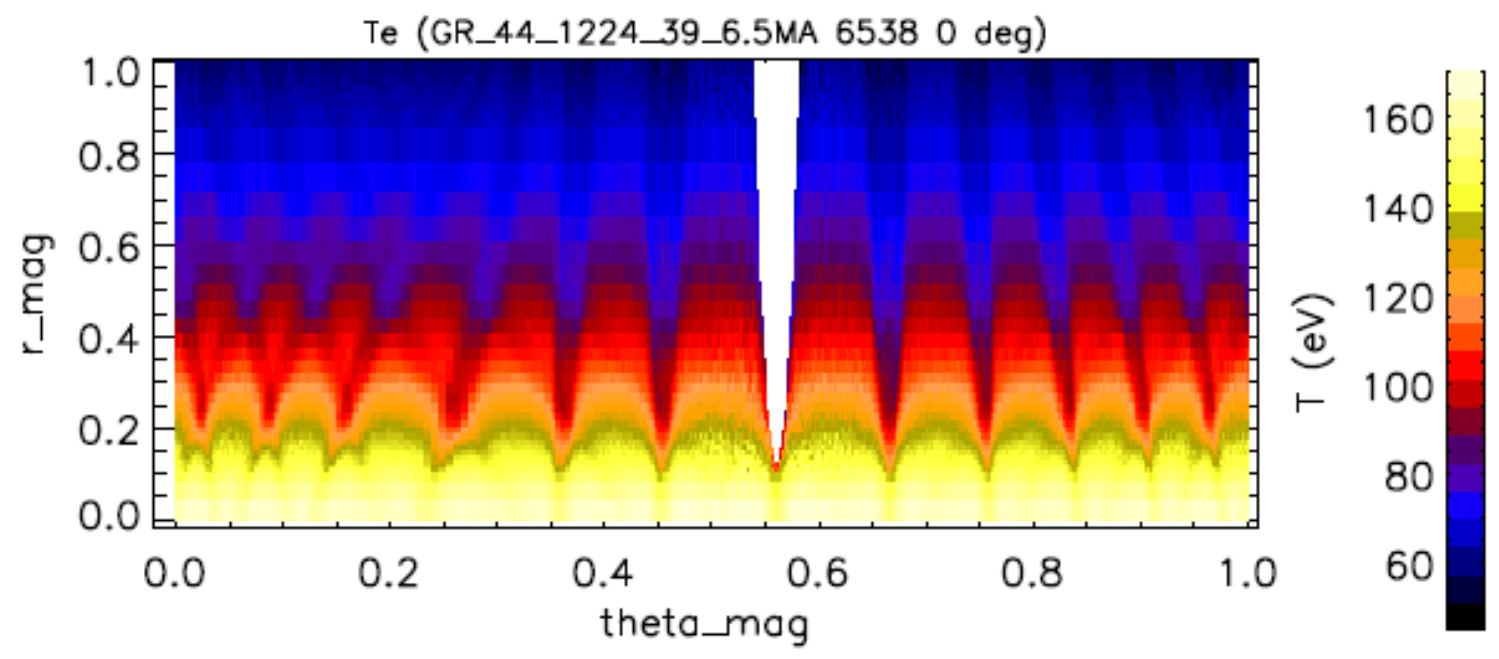

Fig. 8 Te profile in $r_{\text {mag }}$ and $\theta_{\text {mag }}$ coordinates, for a comparison with Fig. 12. The structure in the $\mathrm{L}_{\mathrm{C}}$ profile is not visible except for the shadow region created by main resonance of $\mathrm{q}=6$, where Te becomes small.

\subsubsection{In radial direction}

The collisionality for each flux tube is estimated by,

$$
v^{*}=\frac{s}{\lambda_{e e}},(4-1)
$$

where s and $\lambda_{e e}$ are the distance along flux tube measured from limiter surface and the electronelectron collision mean free path, 


$$
\lambda_{e e}=1.727 \times 10^{17} \frac{T_{e}^{2}}{n \ln \Lambda}(\mathrm{m}),
$$

here $\mathrm{T}_{\mathrm{e}}$ and $\mathrm{n}$ are in $\mathrm{eV}$ and $\mathrm{m}^{-3}$, and $\ln \Lambda$ is typically $\sim 13$. Taking the upstream value for $\mathrm{T}_{\mathrm{e}}$ and $\mathrm{n}, \lambda_{e e}=30 \sim 60 \mathrm{~m}$. For short $\left(2 \mathrm{~s} \sim \mathrm{L}_{\mathrm{C}}=2 \pi \mathrm{Rq}=250 \mathrm{~m}\right)$ and long $(\sim 1700 \mathrm{~m})$ flux tubes, $v^{*}$ is estimated to be $\sim 4$ (collisionless case) and 15 (medium collisional case), respectively. The parallel energy transport time scale, $\tau_{E / /}$, is then obtained for both collisional and collisionless cases as follows,

$$
\begin{gathered}
\tau_{E / /}=\frac{3 n_{u} T_{u} s}{q_{/ /}}=\frac{3 n_{u} T_{u} s}{\frac{4 \kappa_{0} T_{u}^{7 / 2}}{7 s}}=\frac{21 n_{u} s^{2}}{4 \kappa_{0} T_{u}^{5 / 2}}, \text { (collisional case) } \\
\tau_{E / /}=\frac{3 n_{u} T_{u} s}{q_{/ /}}=\frac{3 n_{u} T_{u} s}{\gamma \frac{n_{u}}{2} C_{s} T_{u}}=\frac{6 s}{\gamma C_{s}}, \text { (collisionless case) }
\end{gathered}
$$

where $\kappa_{0}=1.25 \times 10^{22} 1 /\left(\mathrm{m} \mathrm{seV}^{2.5}\right)$, and $\gamma$ and $C_{S}$ are the sheath heat transmission factor $(\sim 8)$ and the ion sound speed at the target, respectively. The subscript $u$ denotes the upstream values. The eqs. (4-3) and (4-4) are plotted in Fig. 9 as a function of $s$ for different $\mathrm{T}_{\mathrm{u}}$. For the short and long flux tubes, one gets $\tau_{E / /} \sim 1 \mathrm{~ms}$ and $6 \mathrm{~ms}$, respectively. On the other hand, the perpendicular energy transport time scale, $\tau_{E \perp}$, is given by,

$$
\tau_{E \perp}=\frac{\Delta r^{2}}{2 \chi_{\perp}}=1 \sim 2 \mathrm{~ms},
$$

with $\chi_{\perp}=1.0 \mathrm{~m}^{2} / \mathrm{s}$ and $\Delta r$ being the SOL width, which is, on average $\sim 5 \mathrm{~cm}$. When , the crossfield transport dominates over parallel transport. As shown in Fig. 9, these conditions are achieved for $\mathrm{s}>\sim 500 \mathrm{~m}$.

Plotted in Fig. 10 are parallel $\mathrm{T}_{\mathrm{e}}$ profiles along the flux tubes for the different $\mathrm{L}_{\mathrm{C}}$ 's, $\sim 250$ and $1700 \mathrm{~m}$. The long flux tube has a higher $\mathrm{T}_{\mathrm{e}} \sim 140 \mathrm{eV}$ at the upstream $\mathrm{s} \sim 1000 \mathrm{~m}$, while the $\mathrm{T}_{\mathrm{e}}$ of the short flux tube is $\sim 110 \mathrm{eV}$ around its upstream $(\mathrm{s} \sim 100 \mathrm{~m})$. The $\mathrm{T}_{\mathrm{e}}$ profile saturates at $\mathrm{s}>500$ $\mathrm{m}$ in Fig. 10 for the long flux tube, where $\tau_{E \perp}<\tau_{E / /}$ is satisfied. This clearly indicates that the energy fed to the flux tube from the core plasma is simply lost radially via cross-field transport, and thus eq. (1-2) does not apply in this region. 


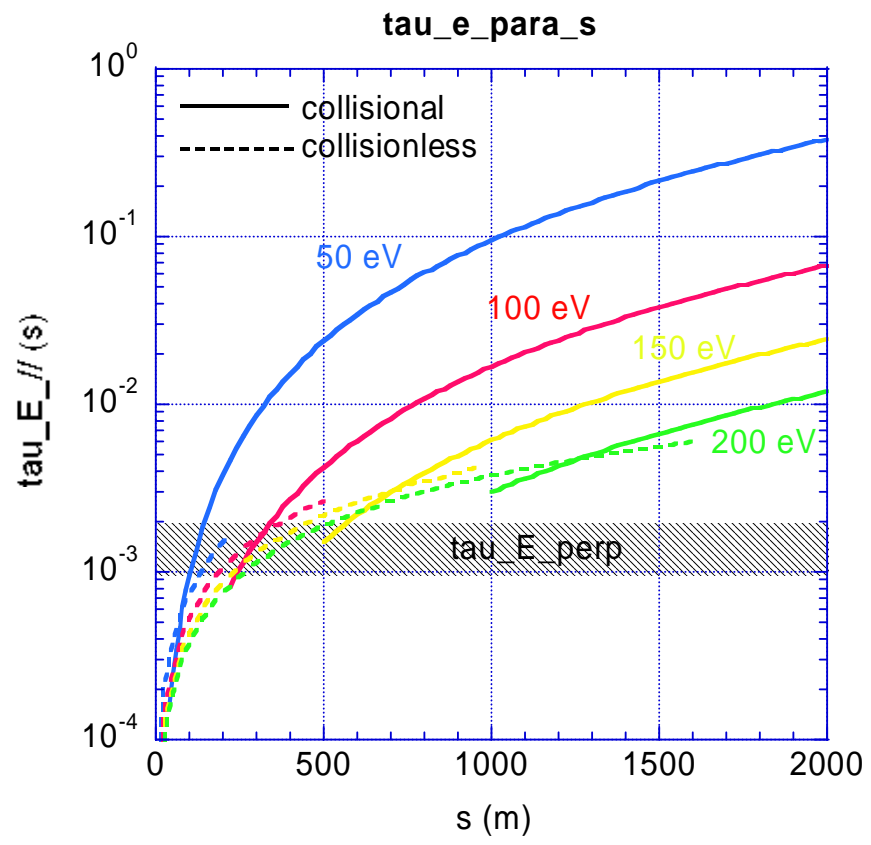

Fig. 9 The parallel energy confinement time, $\tau_{E / /}$, Eqs. (4-3) and (4-4) plotted as a function of $s$, distance along flux tube measured from limiter surface, for different $\mathrm{T}_{\mathrm{u}}$ 's. The perpendicular energy confinement time, $\tau_{E \perp}$, for the $\mathrm{SOL}$ width of $5 \mathrm{~cm}$ is also indicated.

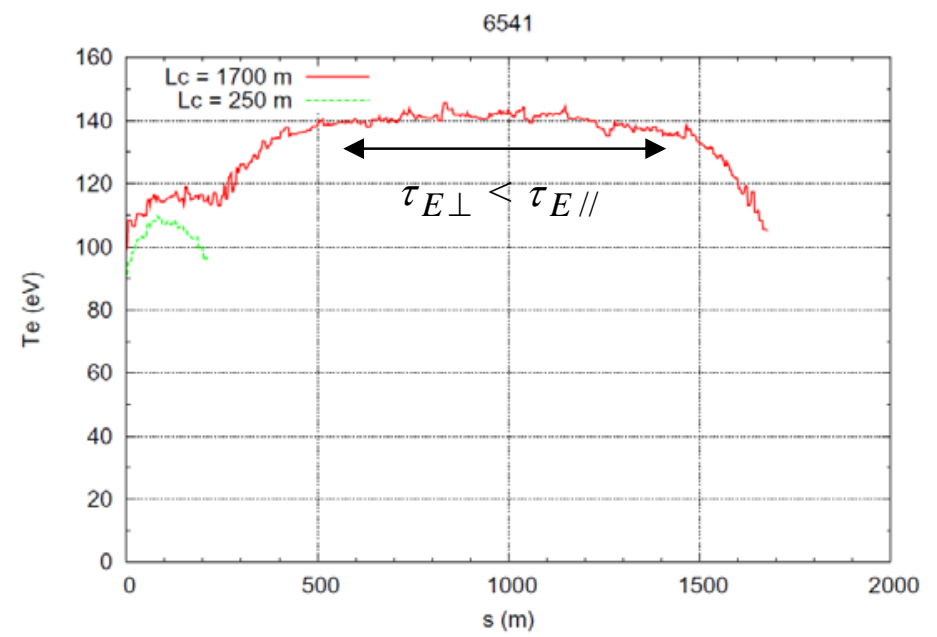

Fig. 10 Parallel $\mathrm{T}_{\mathrm{e}}$ profiles for the different $\mathrm{L}_{\mathrm{C}}$ 's, $\sim 1700$ and $\sim 250 \mathrm{~m}$. $\mathrm{s}$ is measured along the flux tubes, starting from the limiter surface. The short flux tube, $\mathrm{L}_{\mathrm{C}} \sim$ $250 \mathrm{~m}$, travel 6 turns around torus while the longer one, $\sim 1700 \mathrm{~m}, 54$ turns.

For $\mathrm{s}<500 \mathrm{~m}$, where the parallel transport dominates, the flux tubes can still interact significantly with the neighboring ones via cross-field transport within a perpendicular distance of,

$$
\Delta x<\Delta r_{C}=\sqrt{2 \chi_{\perp} \tau_{E / /}} \sim 4 \mathrm{~cm}, \text { (4-6) }
$$

where $\Delta x$ and $\Delta r_{C}$ are the distance between the flux tubes and a characteristic perpendicular transport length. Since the finer structure of $\mathrm{L}_{\mathrm{C}}$ observed in Fig. 3 is a few $\mathrm{mm}$ in radial direction, which is much less than $\Delta r_{C}$, the cross field transport will smear out and it does not appear in the plasma parameter profiles as shown in Fig. 8. 


\subsubsection{In poloidal direction}

In poloidal direction, the poloidal extent of the shadow (short $\mathrm{L}_{\mathrm{C}}$ region at the resonance surface) is of the order of $1 \mathrm{~m}$. They are separated the long (in fact, infinity exactly at the rational surfaces) flux tubes in-between. At these flux surfaces, therefore, the poloidal cross field transport is only the way to carry the energy to this region, which is too small to smear out the pattern. This is the reason for the shadow regions to appear in the $T_{e}$ profile.

Between the resonance (q-rational) surfaces, the flux tubes can transit in poloidal direction substantially to fill out the each flux surface before they hit the limiter. The field line pitch relative to the rational surfaces is given as,

$$
\Theta^{*}=\frac{r_{i} a}{R} \frac{d}{d r}\left(\frac{1}{q}\right),
$$

where $r_{i}$ is a distance from the rational surface. Since in this configuration,

$$
\Theta^{*} \sim 2 \times 10^{-3}>>\sqrt{\frac{\chi_{\perp}}{\chi_{/ /}}} \sim 10^{-4},
$$

the parallel transport is much more effective to distribute the energy in the poloidal direction than the cross field one.

While travelling in the poloidal direction, the flux tubes transfer the energy to the short ones when they pass through the shadow regions, i.e. in the radial direction via cross field transport as explained above. This is clearly seen in Fig. 10 at s $<500 \mathrm{~m}$, where the distance between the flux tubes becomes small such that the condition of eq. (4-6) is satisfied and $T_{e}$ of the long flux tube starts to decrease. At the limiter surface, the temperature becomes almost the same, $\sim 100 \mathrm{eV}$. Consequently, there exists no large difference of the energy deposition of the two flux tubes. In Fig. 8 , it is noted that the radial scale is $\sim$ several $\mathrm{cm}$ on a poloidal average (because of the flux expansion, it becomes wider around top/bottom and narrow around the mid-plane, as seen in Fig. 2.). Thus the radial distance between the rational surfaces is $\sim 1.5 \mathrm{~cm}$ at the LFS midplane.

The energy deposited onto the limiter by the flux tubes with different $\mathrm{L}_{\mathrm{C}}$ 's is plotted in Fig. 11, together with the area of the cross section of the flux tubes. The values are normalized to the total energy and area, respectively. The figure shows that the energy is almost proportional to the area or even slightly distributed more to the shorter ones $(\sim 200 \mathrm{~m})$. This indicates that the cross field transport is effective enough to transfer the energy to the shorter flux tubes. The transport process described above is illustrated in Fig. 12. 


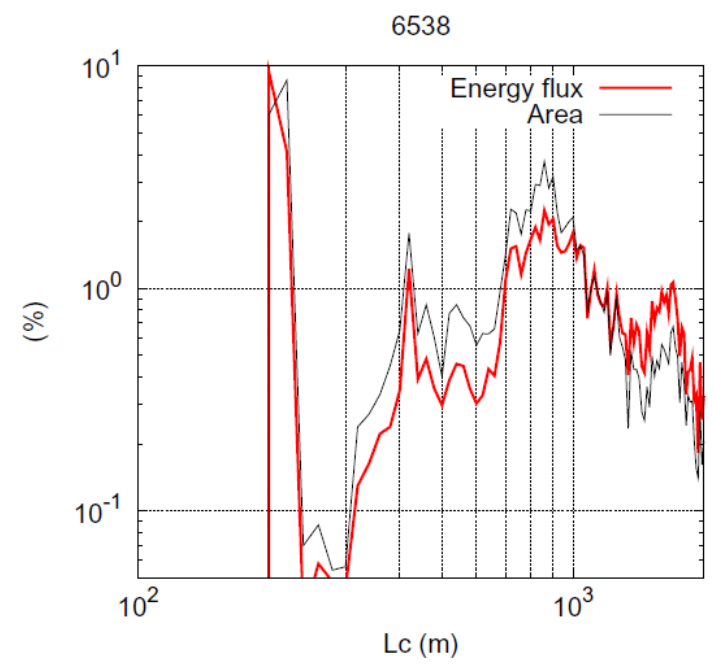

Fig. 11 The energy deposited onto the limiter by the flux tubes with different $\mathrm{L}_{\mathrm{C}}$ 's, together with the area of cross section of the flux tubes. The values are normalized to the total energy and area, respectively.

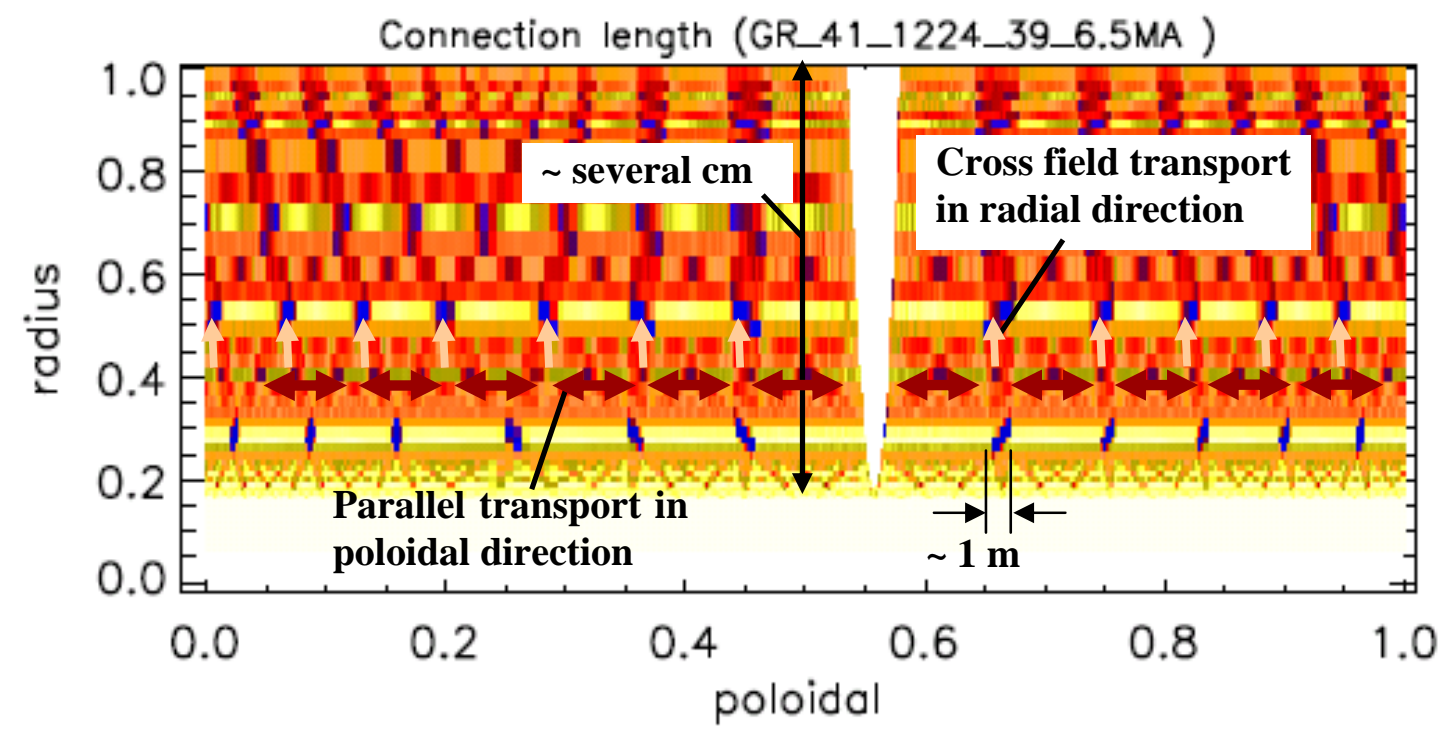

Fig. 12 The transport process is illustrated with the arrows at $\mathrm{r}_{\mathrm{mag}} \sim 0.4$, on the $\mathrm{L}_{\mathrm{C}}$ profile with the color scale being same as Fig. 3 (c). The vertical arrows represent the radial transport which brings the energy from long flux tubes to short ones at the shadow region. The horizontal arrows represent the parallel transport in poloidal direction between the rational surfaces. In addition, there is also energy input from core plasma across the LCFS, which is not indicated in the figure. It is noted that the radial extent of the plot is $\sim$ several $\mathrm{cm}$, the poloidal extent of the shadow is $\sim 1 \mathrm{~m}$. The same transport process applies at the different flux surfaces, i.e. $r_{\text {mag }} \sim 0.2,0.65$ etc.

The transport process in the toroidally discrete limiter configuration is summarized as follows.

1. For the long flux tubes, at $\mathrm{s}>\sim 500 \mathrm{~m}, \tau_{E \perp}<\tau_{E / /}$, the cross field transport dominates over parallel one, resulting in no net energy input.

2. When eq.(4-6) is satisfied, $\Delta x<\Delta r_{C}=\sqrt{2 \chi_{\perp} \tau_{E / /}}$, there exists strong interaction between the flux tubes, giving rise to an energy transfer from long flux tubes to short ones. 
3. This two effects reduce the dependence of eq. (1-2), $\Gamma_{/ /} \propto \sqrt{L_{C}}$, significantly. This is the reason why no localized power load due to the long flux tubes is observed in this configuration.

\subsubsection{Possibility of localized power deposition}

If the distance between the long and short flux tubes are comparable with $\Delta r_{C}$, i.e. $\Delta x \sim \Delta r_{C}$, the long flux tube can keep high energy flux up to the limiter surface without the smearing effect via cross-field transport. At Ip $=2.5 \mathrm{MA}$, because of the low shear of q (Fig. 4), the distance between the resonance surface becomes large, $\sim 7 \mathrm{~cm}$. Figure 13 shows an example of the localized power deposition, where $\mathrm{L}_{\mathrm{C}}$ profile on the limiter is plotted together. It is seen that separated deposition pattern appears around $(\mathrm{Y}, \mathrm{Z})=(-0.7,0.9)$ and $(0.7,0.6) \mathrm{m}$ where the long flux tubes hit the limiter, with a substantial size in radial direction, $\sim 3.5 \mathrm{~cm}$. In Fig. 13 (b) the region of the long flux tube extends towards the center of the limiter $(\mathrm{Y}=0 \mathrm{~m})$, but due to the decreasing incidence angle, the deposition becomes small. In this plasma current, however, because of $\mathrm{P}_{\mathrm{SOL}}(=2.0 \mathrm{MW})$ the peak value is fortunately not so high $\left(<2.0 \mathrm{MW} / \mathrm{m}^{2}\right)$. Nevertheless, the result shows that the treatment of the 3D geometry of the flux tubes can be important for detailed analysis of the power deposition for determining critical design value.
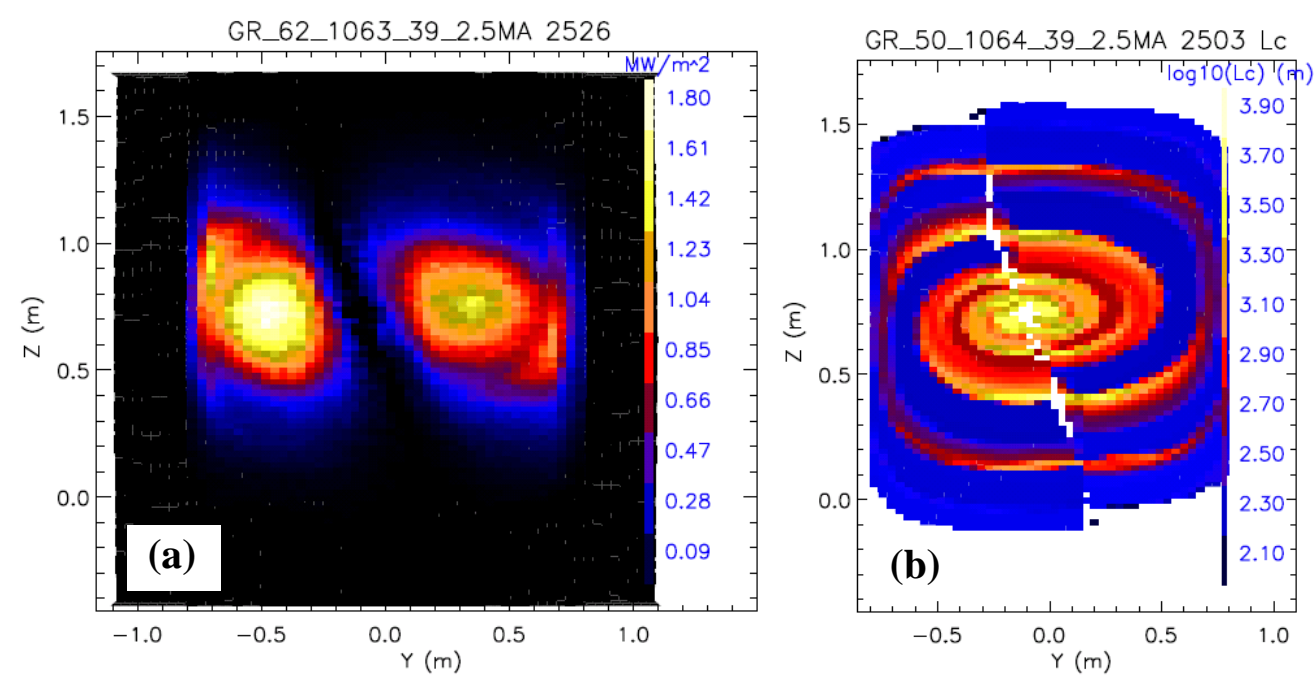

Fig. 13 (a) Power deposition profile (b) $\mathrm{L}_{\mathrm{C}}$ profile on the limiter surface, for $\mathrm{Ip}=2.5 \mathrm{MA}, \mathrm{P}_{\mathrm{SOL}}=2.0 \mathrm{MW}, D_{\perp}=\chi_{\perp}=0.7 \mathrm{~m}^{2} / \mathrm{s}$. The localized deposition pattern appears around $(\mathrm{Y}, \mathrm{Z})=(-0.7,0.9)$ and $(0.7,0.6) \mathrm{m}$ where the long flux tubes hit the limiter, with a substantial size in radial direction, $\sim 3.5 \mathrm{~cm}$.

\section{Parameter scan}

\subsection{Choice of diffusion coefficients for ITER start-up phase}

In the present analysis, the perpendicular transport coefficients, $D_{\perp}$ and $\chi_{\perp}$, are assumed to be anomalous. In order to estimate those for ITER limiter configuration, we have examined the available experimental data and the proposed scalings and have concluded to base our extrapolation on the JET experimental data. The reasons for this are two fold : a) one of the most complete experimental study of edge plasma 
conditions and SOL transport for ohmic limiter plasmas was carried out at JET [8] providing experimental information over a large range of conditions and b) JET being the largest limiter tokamak ever in operation could reach plasma conditions in ohmic limiter discharges very similar to those expected in ITER during the ramp up, including the magnitude of the plasma current of $7 \mathrm{MA}$, except for the plasma size. The experimental studies in [9] and [8] for JET ohmic limiter plasma with $\mathrm{I}_{\mathrm{p}}=1-5$ MA indicate that there the temperature $\left(\lambda_{T}\right)$ and density $\left(\lambda_{n}\right)$ e-folding lengths in the SOL scale with $\mathrm{I}_{\mathrm{p}}^{-1}$ and are weakly depend on plasma density (within the achievable range for each density), particularly $\lambda_{n}$ (similar results are obtained in TEXTOR [10]) as shown in Fig. 14 (from [9]). Typically $\lambda_{T}>\lambda_{n}$, with values in the range $\lambda_{T} / \lambda_{n}=1-2$, with the two e-folding lengths being similar at lower currents $\left(\mathrm{I}_{\mathrm{p}}=2 \mathrm{MA}\right)$. Such values of the ratio $\lambda_{\mathrm{T}} / \lambda_{\mathrm{n}}$ can be related to a ratio of the particle and energy diffusion coefficients' in the range $\chi_{\perp} / D_{\perp}=1-5$ for typical SOL plasma conditions with low levels of local re-ionisation (typically $\sim 25 \%$ of the recycled ion flux) as seen from TEXTOR experiments [10], which is also found in modelling of diverted L-mode discharges of ASDEX-Upgrade [11]. The observed dependence of $\lambda_{n}$ and $\lambda_{T}$ with $\mathrm{I}_{\mathrm{p}}$ is reflected into a dependence of $\chi_{\perp} \& D_{\perp} \sim \mathrm{I}_{\mathrm{p}}^{-\alpha}$, with $\alpha=1.5-2.0$, which is also found by edge modelling of diverted discharges in ASDEX-Upgrade [11]. In particular the derived scaling for $D_{\perp}$ from [8] (see Fig. 15) is given approximately by

$$
D_{\perp}\left(\mathrm{m}^{2} \mathrm{~s}^{-1}\right)=\beta \times 9.0 \mathrm{I}_{\mathrm{p}}(\mathrm{MA})^{-2}
$$

where $\beta$ accounts for the variation of the coefficient with plasma density at constant plasma current and is $\beta=1 \pm 0.5$.
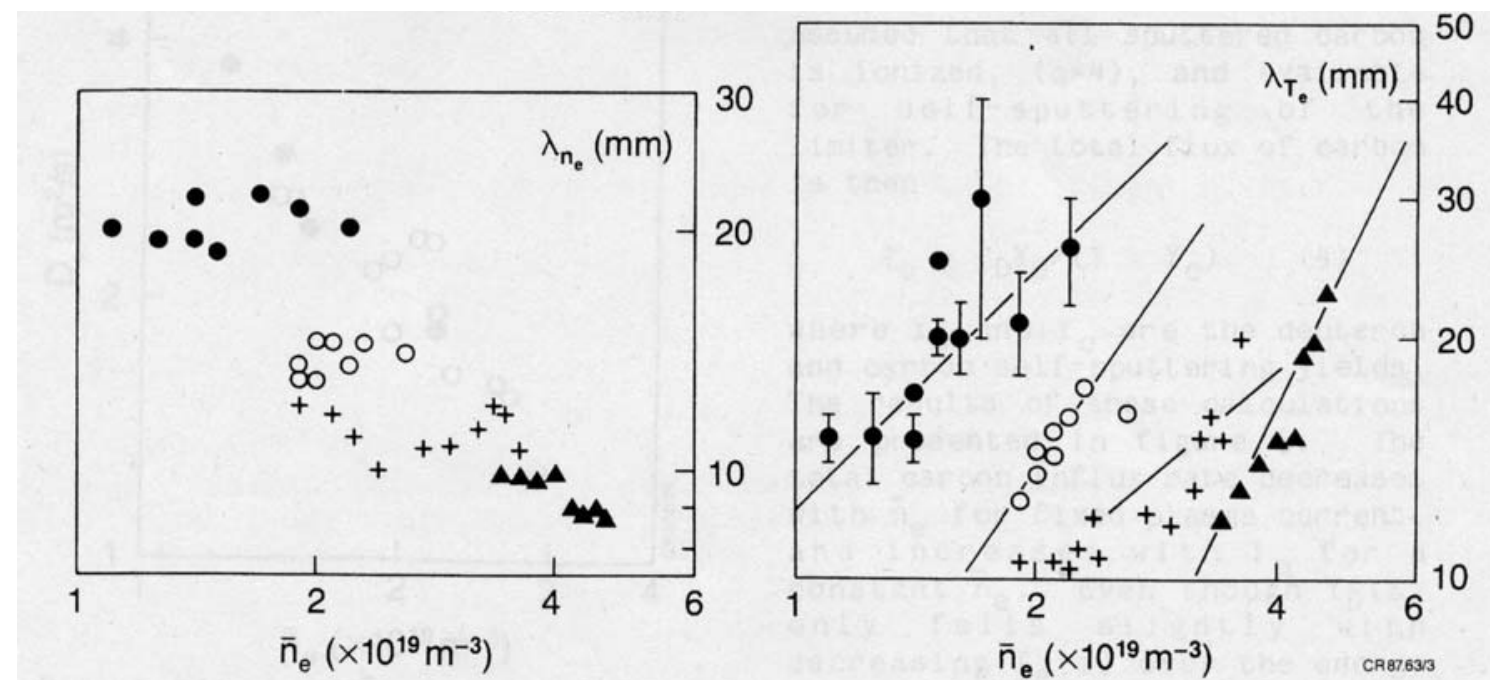

Fig. 14 The effect of $\bar{n}_{e}$ and $\mathrm{I}_{\mathrm{p}}$ on density $\lambda_{\mathrm{n}}$ and temperature $\lambda_{\mathrm{T}}$ e-folding length in the SOL of JET limiter discharge. $($ closed circle $=2 \mathrm{MA}, 2.1 \mathrm{~T}$; open circle $=$ $3 \mathrm{MA}, 3.4 \mathrm{~T}$; cross $=4 \mathrm{MA}, 3.4 \mathrm{~T}$; triangle $=5 \mathrm{MA}, 3.4 \mathrm{~T}$ ) [9] 


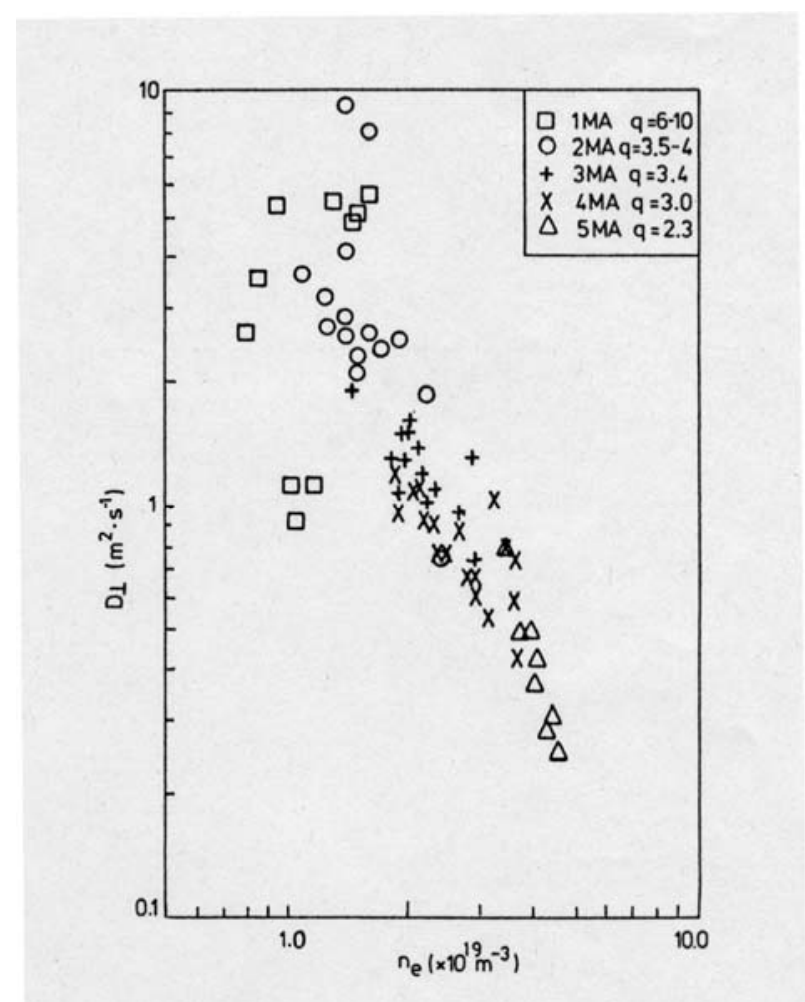

Fig. 15 Cross-field diffusion rate $D_{\perp}$ calculated from the density e-folding length in JET limiter discharge [8].

In order to determine the values of the diffusion coefficients in ITER, besides the above dependences, the size scaling of these coefficients should be determined by comparing measurements from various devices. In general, it is seen that the radial diffusion coefficients scale weakly with device size [12] for low field tokamak devices. Studies of SOL multi-machine thickness scalings for ohmic limiter discharges do not exist. The size scaling of the transport coefficients must be thus derived from the existing databases for low recycling diverted discharges in ohmic or L-mode conditions for which edge SOL transport is expected to be comparable to limiter discharges. Existing scalings of the SOL thickness for such discharges reveal the dependencies $\lambda \sim \mathrm{R}^{1.2} \mathrm{I}_{\mathrm{p}}{ }^{-0.7}[13]$, which correspond to a dependence of $\mathrm{D}(\& \chi) \sim \mathrm{R}^{1.4}$ $\mathrm{I}_{\mathrm{p}}{ }^{-1.4}$. Given the uncertainties in the determination of this size dependence and the similarities of the multi-machine L-mode diverted discharges scaling with that derived from JET limiter discharges, it seems prudent to take for modelling of ITER that from JET, which leads to the choice of diffusion coefficients for our studies summarised in Table 1. It is important to note that the increase of diffusion coefficient which could be associated with such size scaling is of the same order of the variation of diffusion coefficients with density which is included in $\beta$. Furthermore, our prescription would lead in the worse case to an over estimate of the power loads on the ITER limiter by a factor of $\sqrt{2.7}$ which is well within the observed ion-electron drift-side limiter asymmetries, which are not modelled in this study. 


\subsection{Parameter scan}

We have selected three cases for the combination of the up-stream density and $\mathrm{P}_{\mathrm{SOL}}$ based on the study of Ref. [7] The parameter range is summarized in table. 1, together with $D_{\perp}$ 's, which were scaled from the JET data with respect to the plasma current. The third case, $0.5 \mathrm{n}_{\mathrm{G}}$ with $+50 \% \mathrm{P}_{\mathrm{SOL}}$, was chosen to cover the uncertainty discussed in [7]. In order to speed up the computations, only the energy transport equations were analyzed, while the density profile was given assuming a certain decay length. The procedure for the computation is as follows.

Because of the expected longer connection lengths in ITER than that in JET and also because of the different magnetic field structure (due to the different $\mathrm{q}_{\text {edge }}$ and the limiter configuration) as shown in section 3 , the absolute values of the decay length of density $\left(\lambda_{\mathrm{n}}\right)$ and electron temperature $\left(\lambda_{\mathrm{Te}}\right)$ will be different for the ITER configuration. Therefore, we simply scale the ratio of the decay lengths, $\lambda_{\mathrm{Te}} / \lambda_{\mathrm{n}}$. Also from the JET experiments, we have found that the perpendicularly convected energy flux is within the range from $30 \%$ to $80 \%$ of $\mathrm{P}_{\mathrm{SOL}}$, which provides a condition to define $\lambda_{\mathrm{n}}$. The iterations for the parameter scan then proceeds as follows:

1. Start with the guessed density profile with a certain $\lambda_{\mathrm{n}}$.

2. After a convergence, obtain the results for $\lambda_{\mathrm{Te}}$ and convected power $\mathrm{P}_{\text {conv }}$.

3. Change the $\lambda_{\mathrm{n}}$ and/or $\chi_{\perp}$ within the range of $2 \sim 4 D_{\perp}$ for the next iteration. Repeat the step $1 \sim 3$ until reach the desired $\lambda_{\mathrm{Te}} / \lambda_{\mathrm{n}}$ and $\mathrm{P}_{\text {conv }}$.

As shown in the section 4, however, the radial profiles of the plasma parameters are not simple exponential function. In the present analysis, for the measure of the width of the SOL, the distance for the $T_{e}$ to decrease by a factor of $1 / \mathrm{e}$ of the up-stream value was taken as a decay length.

The resulting decay lengths of parallel energy flux $\left(\lambda_{\mathrm{p}}\right)$, Te $\left(\lambda_{\mathrm{Te}}\right)$ and density $\left(\lambda_{\mathrm{n}}\right)$ are plotted in Fig. 16, together with the peak power values. For the higher Ip (6.5 MA), the decay lengths decrease because of the smaller transport coefficients. The peak power is close to the engineering limit $\left(8 \mathrm{MW} / \mathrm{m}^{2}\right)$ at Ip $=6.5 \mathrm{MA}$ for the case of $0.5 \mathrm{n}_{\mathrm{G}}$ with $+50 \% \mathrm{P}_{\mathrm{SOL}}$. It is found that $\lambda_{\mathrm{n}}$ and $\lambda_{\mathrm{Te}}$ do not follow the simple relation,

$$
\lambda_{n} \propto \sqrt{D_{\perp} L_{C}}, \quad \lambda_{T e} \propto \sqrt{\chi_{\perp} L_{C}},
$$

but rather decrease faster than expected from this correlation going to higher Ip. This is considered due to the higher temperature with increasing $\mathrm{P}_{\mathrm{SOL}}$ at high Ip, which gives rise to a larger parallel conductivity and parallel flow velocity, resulting in a shorter radial decay. The shape of flux surface could be also the reason, i.e. at higher Ip the radial distance of the flux surfaces becomes small at the LFS midplane, which will give shorter decay length there. 
Table. 1 The parameter range to be scanned, for three density cases, $<\mathrm{n}>=0.2 \mathrm{n}_{\mathrm{G}}, 0.5 \mathrm{n}_{\mathrm{G}}$ and $0.5 \mathrm{n}_{\mathrm{G}}$ with $+50 \% \mathrm{P}_{\mathrm{SOL}}$, which were chosen from the core transport simulation study for the ITER startup configuration [7]. $<\mathrm{n}>$ is $80 \%$ of the line averaged density and $\mathrm{n}_{\mathrm{G}}$ is the Greenwald density, respectively. The up-stream density, $\mathrm{n}_{\mathrm{up}}$, was obtained as $20 \%$ of $<\mathrm{n}>$. $D_{\perp}$ is scaled from the JET limiter discharge with respect to the plasma current, according to eq.(5-1). The ration of the decay length of $\mathrm{T}_{\mathrm{e}}$ and $\mathrm{n}$ was obtained from the JET data.

$<0.2 * n+G>$

\begin{tabular}{|c|c|c|c|c|}
\hline Ip (MA) & $\begin{array}{l}\text { Psol } \\
\text { (MW) }\end{array}$ & $\begin{array}{l}\text { D_perp } \\
\left(m^{* *} 2 / s\right)\end{array}$ & $\lambda T / \lambda n$ & $\underset{\left(e 19 m^{* *}-3\right)}{\text { n_up }}$ \\
\hline 2.5 & 1.0 & $1.0,2.0,3.0$ & 1 & 0.12 \\
\hline 4.5 & 2.0 & $0.3,0.65,1.0$ & 1.5 & 0.17 \\
\hline 6.5 & 3.0 & $0.2,0.3,0.4$ & 1.5 & 0.22 \\
\hline \multicolumn{5}{|l|}{$<0.5^{*} n_{-} G>$} \\
\hline Ip (MA) & $\begin{array}{l}\text { Psol } \\
(\mathrm{MW})\end{array}$ & $\begin{array}{l}\text { D_perp } \\
\left(\mathrm{m}^{* *} 2 / \mathrm{s}\right)\end{array}$ & $\lambda T / \lambda n$ & $\underset{\left(e 19 m^{* *}-3\right)}{\text { n_up }}$ \\
\hline 2.5 & 1.3 & $1.0,2.0,3.0$ & 1 & 0.30 \\
\hline 4.5 & 2.6 & $0.3,0.65,1.0$ & 1.5 & 0.44 \\
\hline 6.5 & 4.0 & $0.2,0.3,0.4$ & 1.5 & 0.54 \\
\hline
\end{tabular}

$<0.5 * n \_G>$

$+50 \%$

\begin{tabular}{|c|c|c|c|c|}
\hline Ip (MA) & $\begin{array}{l}\text { Psol } \\
\text { (MW) }\end{array}$ & $\begin{array}{l}\text { D_perp } \\
\left(m^{* *} 2 / s\right)\end{array}$ & $\lambda T / \lambda n$ & $\underset{\left(e 19 m^{* *}-3\right)}{n \_u p}$ \\
\hline 2.5 & 2.0 & $1.0,2.0,3.0$ & 1 & 0.30 \\
\hline 4.5 & 4.0 & $0.3,0.65,1.0$ & 1.5 & 0.44 \\
\hline 6.5 & 6.0 & $0.2,0.3,0.4$ & 1.5 & 0.54 \\
\hline
\end{tabular}



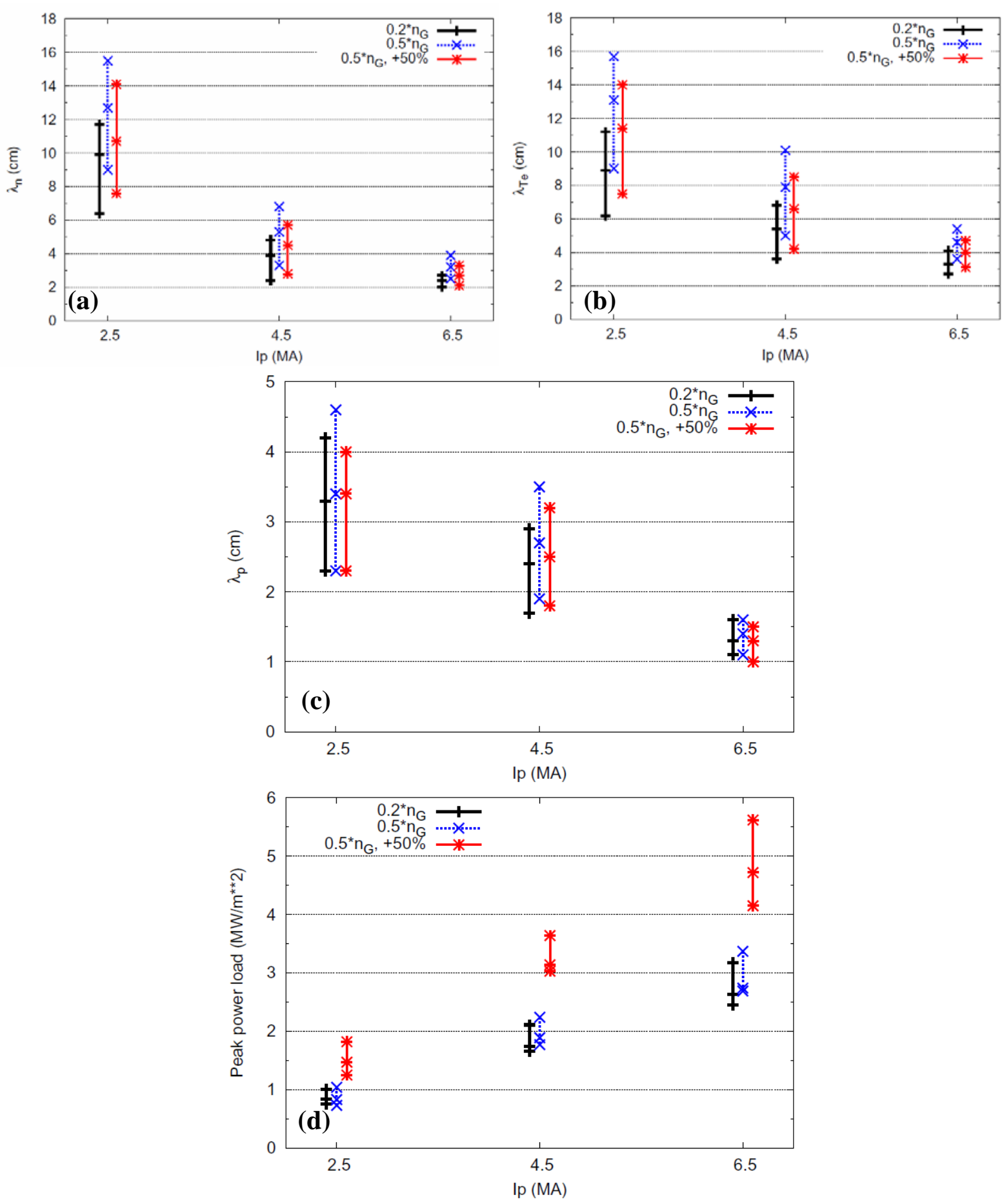

Fig. 16 Results of the parameter scan, (a) $\lambda_{\mathrm{n}}$, (b) $\lambda_{\mathrm{Te}}$, (c) $\lambda_{\mathrm{p}}$ and (d) the peak power load on the limiter surface for the three cases, $0.2 \mathrm{n}_{\mathrm{G}}, 0.5 \mathrm{n}_{\mathrm{G}}$ and $0.5 \mathrm{n}_{\mathrm{G}}$ with $+50 \% \mathrm{P}_{\mathrm{SOL}}$. The peak power load is close to the engineering limit $\left(\sim 8 \mathrm{MW} / \mathrm{m}^{2}\right)$ at $0.5 \mathrm{n}_{\mathrm{G}}$ with $+50 \% \mathrm{P}_{\mathrm{SOL}}$. 

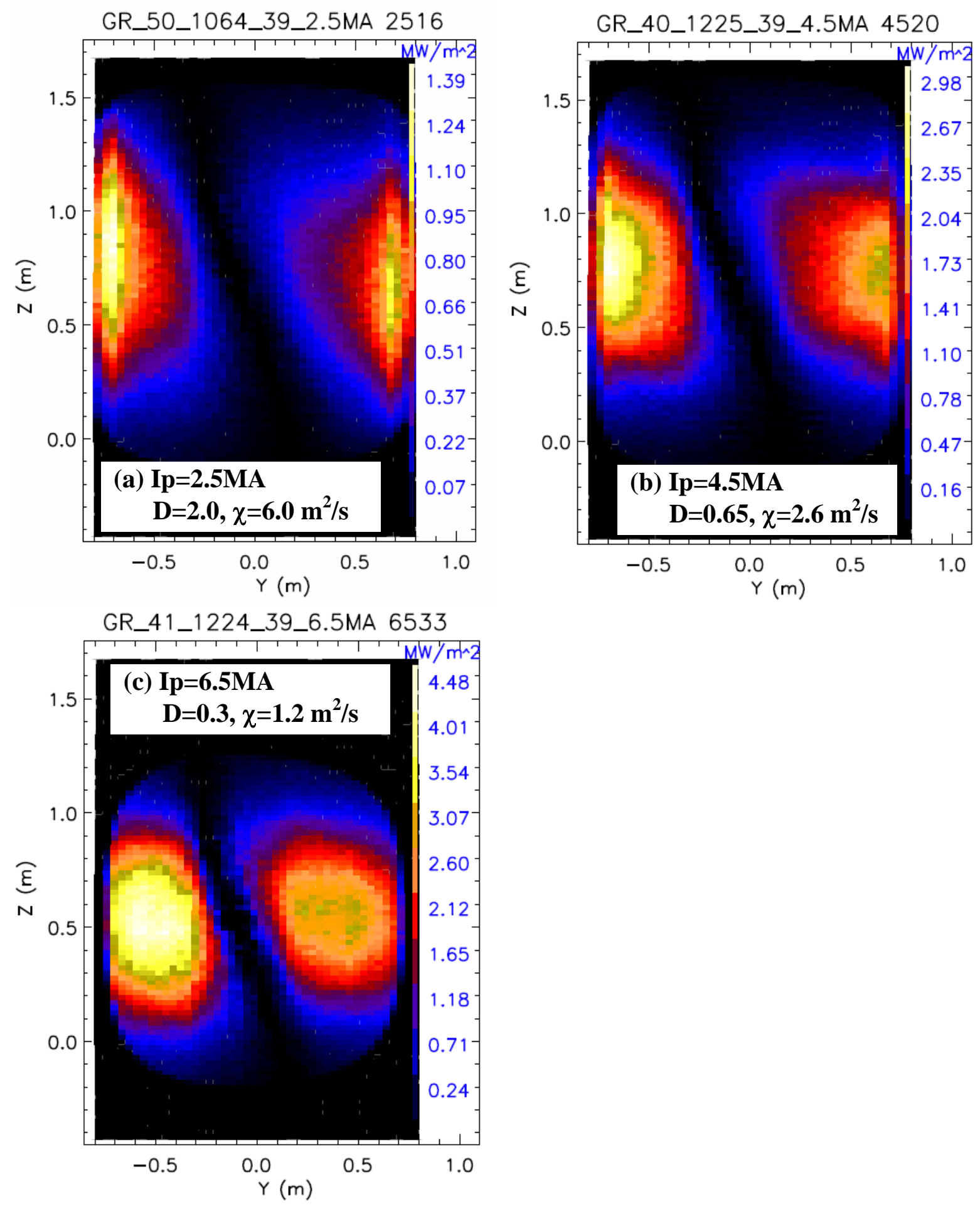

Fig. 17 The power deposition profiles on the limiter surface for the different Ip's with the case of $0.5 * \mathrm{n}_{\mathrm{G}}+50 \% \mathrm{P}_{\mathrm{SOL}}$. (a) Ip=2.5MA, $\mathrm{D}=2.0, \chi=6.0 \mathrm{~m}^{2} / \mathrm{s}$, (b) Ip=4.5MA, $\mathrm{D}=0.65, \chi=2.6 \mathrm{~m}^{2} / \mathrm{s}$, (c) Ip=6.5MA, D=0.3, $\chi=1.2 \mathrm{~m}^{2} / \mathrm{s}$. For the low Ip's, 2.5 and $4.5 \mathrm{MA}$, because of the higher transport coefficients, i.e. larger $\lambda_{\mathrm{p}}$, the power tends to deposit at the edge of the limiter. 
The power deposition profiles are plotted in Fig. 17, for the different Ip's with the case of $0.5^{*} n_{G}$ $+50 \% \mathrm{P}_{\mathrm{SOL}}$. For the lower current configurations, i.e. 2.5 and 4.5 MA, the peak appears at the edge of the limiter due to the long $\lambda_{\mathrm{p}}$. However, because of the small $\mathrm{P}_{\mathrm{SOL}}$, the peak value does not increase as much as for the case with 6.5 MA.

The results suggest that the limiter shape has to be optimized to the higher Ip, because of the higher $\mathrm{P}_{\mathrm{SOL}}$ and of the shorter $\lambda_{\mathrm{p}}$ due to the smaller $D_{\perp} \& \chi_{\perp}$.

\section{Comparison of 3D model with simple model}

Because of the shadow region, there appears also a decay of $\Gamma_{/ /}$in poloidal direction, as shown in Fig. 18, where the poloidal profiles of $\Gamma_{/ /}$is plotted at the different radial positions (the distance from the LCFS). This will create a difference in the deposition pattern from a simple model (e.g. [14]), which assumes only radial decay of $\Gamma_{/ /}$as,

$$
\Gamma_{/ /}=\Gamma_{0} \exp \left(-r / \lambda_{p}\right)
$$

Giving the $\lambda_{p}$ estimated from the 3D modelling and the corresponding total energy deposition $\left(\mathrm{P}_{\mathrm{SOL}}=6.0 \mathrm{MW}\right)$, eq. $(6-1)$ gives the profile shown in Fig. 19. The simple model has larger $\Gamma_{/ /}$at the smaller radius, which is compensated by the slow decay of the $3 \mathrm{D}$ model at the larger radius. In the poloidal direction, an uniform profile is assumed. The resulting deposition patterns are shown in Fig. 20 , together with horizontal and vertical cut of the deposition profiles. One sees that the simple model gives higher peak power load than the $3 \mathrm{D}$ one, due to the uniform poloidal profile of $\Gamma_{/ /}$. As shown in Fig. 20 (c) and (d), the 3D model distributes the energy to the larger area, due to the poloidal decay of $\Gamma_{/ /}$, which is caused by the 3D geometrical effect. In this case, the simple model overestimates the peak power load by $30 \%$. Despite the limitations of this simplified model, which in part have been clearly identified by cross-checking its results with those of the approach described here, it is felt that none the less this is especially suited for parametric analysis and design optimisation studies. A detailed description of this model and the calculation methodology is described elsewhere [14].

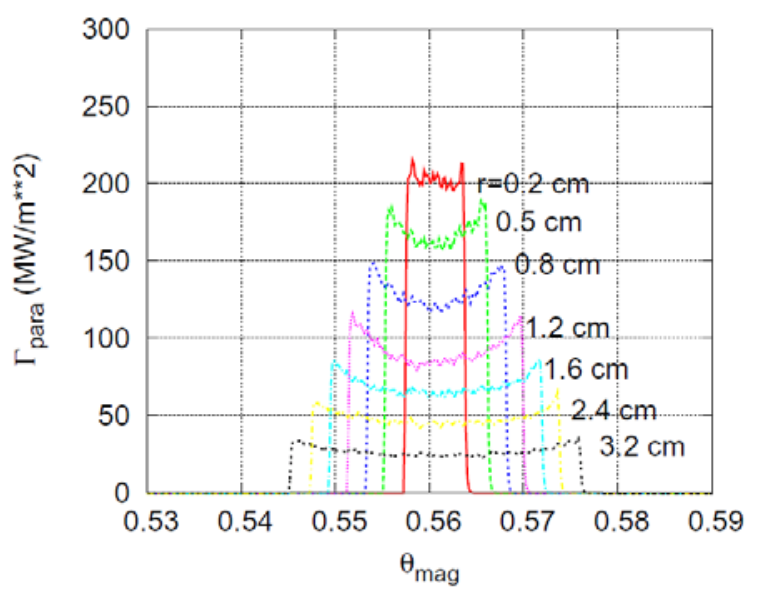

Fig. 18 Poloidal profiles of the parallel energy flux, $\Gamma_{/ /}$obtained by the 3D modelling (EMC3EIRENE), at the different radial position (distance from the LCFS) (Ip $=6.5 \mathrm{MA}, D_{\perp}=0.2$, $\left.\chi_{\perp}=0.8 \mathrm{~m}^{2} / \mathrm{s}, 0.5 \mathrm{n}_{\mathrm{G}}\right)$. There appears decay in the poloidal direction. 

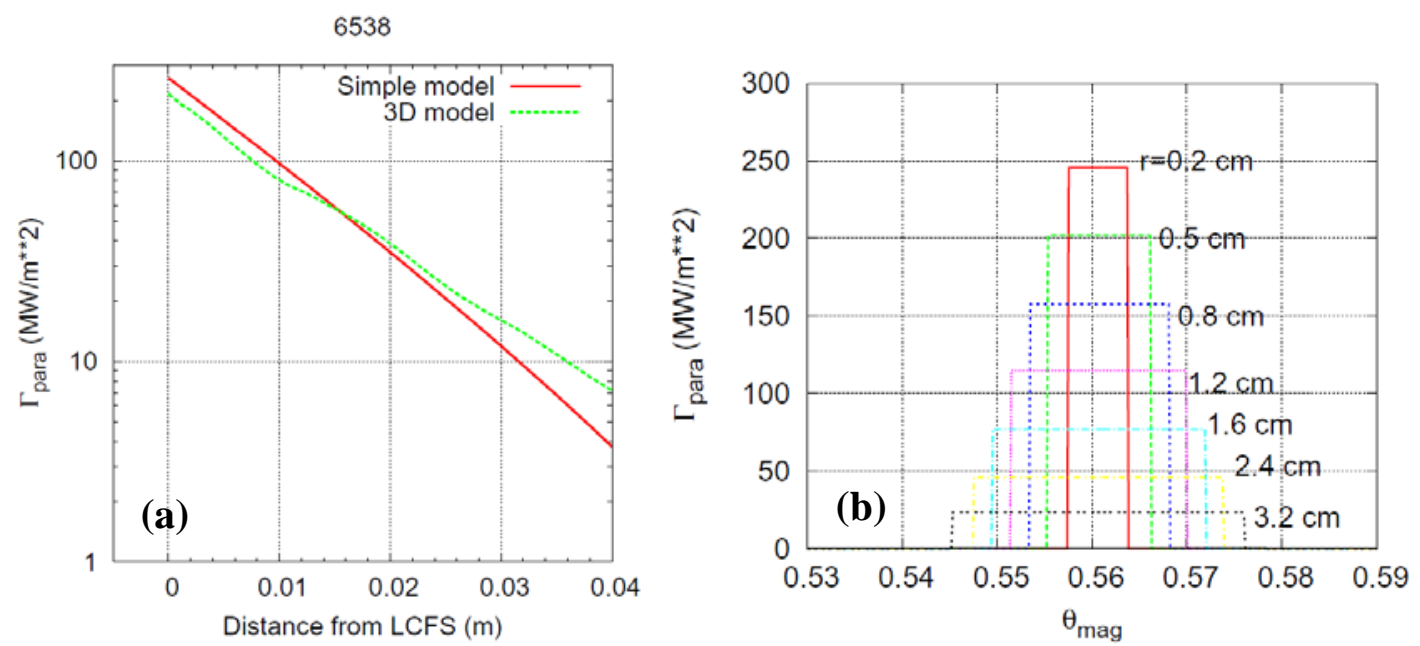

Fig. 19 (a) Radial profiles of $\Gamma_{/ /}$obtained by the 3D model together with the simple model, eq. (61). (b) Poloidal profile of $\Gamma_{/ /}$for the simple model, which assumes uniform $\Gamma_{/ /}$in the poloidal direction. The simple model uses $\lambda_{\mathrm{p}}$, which is estimated from the $3 \mathrm{D}$ model.
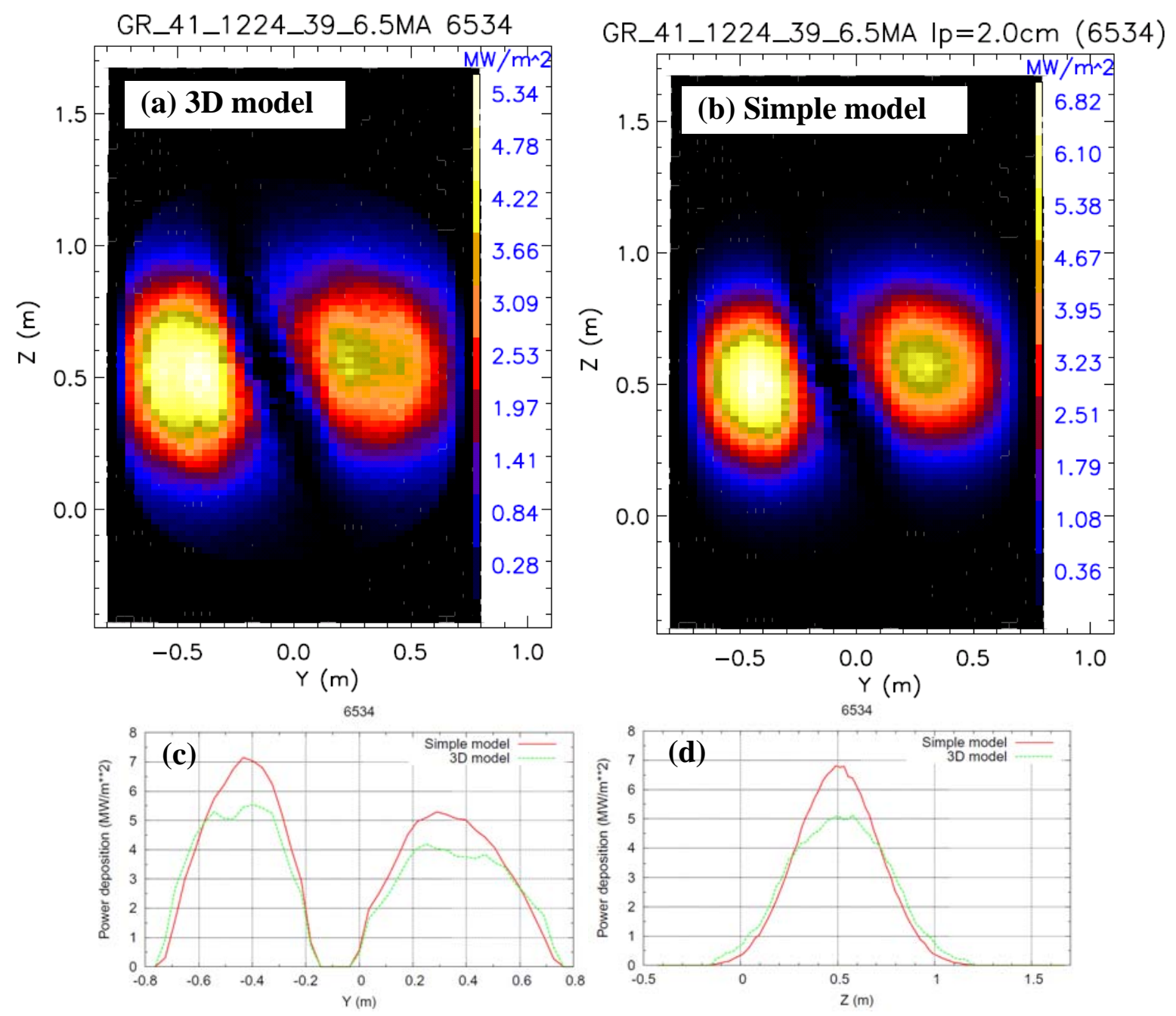

Fig. 20 Power deposition profiles of (a) the 3D model and (b) the simple model, obtained from the $\Gamma_{/ /}$in Fig. 19. (c) horizontal cut of the deposition profile at $Z=0.5 \mathrm{~m}$. (d) vertical cut at $Y=$ $-0.5 \mathrm{~m}$. The simple model uses $\lambda_{\mathrm{p}}$, which is estimated from the $3 \mathrm{D}$ model. 
Figure 21 shows the dependence of the peak power load on $\lambda_{p}$ for both the $3 \mathrm{D}$ and simple models, with $\mathrm{P}_{\mathrm{SOL}}=6.0 \mathrm{MW}$. Less than $\lambda_{p}=1.5 \mathrm{~cm}$, the peak power load starts to increase significantly in the simple model, which is in agreement with the previous assessment. The increase in the $3 \mathrm{D}$ model is, however, modest. For the case with density \& momentum equations, due to the increase of the density in front of the limiter, the energy is deposited more to the centre of the limiter, where the incidence angle of flux tubes is small. It effectively reduces the peak power load for the same $\lambda_{p}$ as seen in Fig. 21. The result shows that the simple model with an exponential radial decay tends to overestimate the peak power load on the limiter.

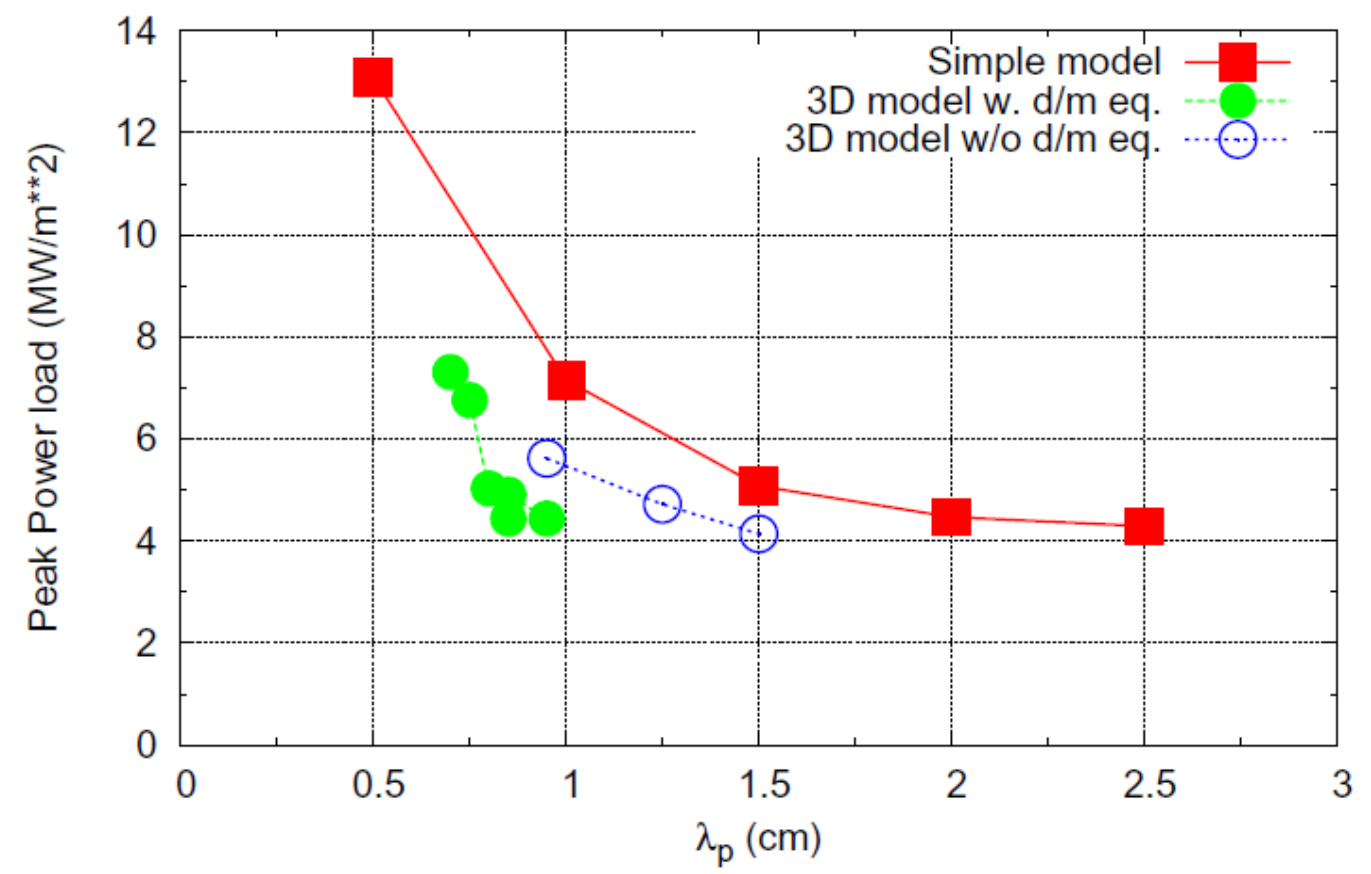

Fig. 21 Peak power load on the limiter as a function of $\lambda_{p}$ for $\mathrm{Ip}=6.5 \mathrm{MA}, \mathrm{P}_{\mathrm{SOL}}=6.0 \mathrm{MA}$, with 3D and simple models. Square : simple model eq.(6-1), closed circle : 3D model with density \& momentum equations, open circle : 3D model without density \& momentum equations. The simple model uses $\lambda_{\mathrm{p}}$, which is estimated from the $3 \mathrm{D}$ model.

\section{Discussion and recommendations for further work}

In the present 3D model, the power deposition onto the limiter surface is given by only parallel transport through Bohm boundary condition. In the experiments, there will be also perpendicular deposition, and we will have a certain amount of power load at the center of the limiter despite zero incidence angle. Nevertheless, peak power load is expected to appear at the off-center region as shown in the present analysis, like the experimental results in existing tokamaks [15].

The determination of engineering limit is still under discussion and the value referred in this report, $8 \mathrm{MW} / \mathrm{m}^{2}$, can be lowered in future. In that case, taking into account of the highest power load obtained in the $3 \mathrm{D}$ results, $\sim 6 \mathrm{MW} / \mathrm{m}^{2}$, it may be necessary to switch to the divertor configuration at early phase, e.g. at $\mathrm{I}_{\mathrm{p}}=4.5 \mathrm{MA}$, where already X-point is established. 
The study presented here should be extended in several ways and recommendations are made on areas that need improvements.

- In the previous assessment, the toroidally asymmetric limiter shape was obtained in order to accommodate an asymmetry of the power flux depending on the drift direction of electrons and ions, although in the present analysis we have assumed symmetrical power flux in toroidal direction for simplicity. For a final optimization of the limiter shape, however, evaluation of power loads resulting from possible asymmetry of energy in the ion-drift and electron-drift channel will be necessary

- In a real configuration, a toroidal ripple will appear in the magnetic field. This will change an incidence angle of the flux tubes to the limiter surface to a certain extent, resulting in a modification of power deposition pattern. The model has to be extended to include the ripple effect.

- The effect of misalignment of the limiters on a power load asymmetry has to be analyzed, especially for the higher plasma current, e.g. $\mathrm{I}_{\mathrm{p}}=6.5 \mathrm{MA}$, where the asymmetry will be large because of the shorter $\lambda_{\mathrm{p}}$.

- Although we have scanned the transport coefficients within a possible range based on the experiments, it is still necessary to characterise the SOL parameters during limiter start-up, and ramp down in present tokamaks, and to compare them with the values during the flat-top, and with modelling. This would give important data for the design of the ITER start-up limiters.

- In the standard poloidal divertor configurations, formation of radial E-field and its effect on transport have been studied in ref $[16,17]$ in a self-consistent manner.

From the temperature profiles in the limiter SOL obtained by EMC3-EIRENE in the ITER configuration (e.g. Fig. 6 (a)), one would simply expect a radial E-field in the range of $T_{\mathrm{e}} / \lambda_{\mathrm{Te}} \sim 3 \mathrm{kV} / \mathrm{m}$, which results in an ExB drift velocity of about $500 \mathrm{~m} / \mathrm{s}$ for $\mathrm{B}=6 \mathrm{~T}$. The drift time scale for the poloidal limiter shadow of order of $1 \mathrm{~m}$ is therefore ms which is comparable to the SOL transport time as discussed in section 4.2, and may moderate the effect of different connection lengths. In fact, however, the drift is not purely poloidal. Rather, it should more or less follow the temperature contour which is strongly modulated by the limiter shadow. Therefore, a reasonable assessment of the drift effect is only possible after including the drift terms in the 3D code, which will be a further work of this task.

\section{Conclusions}

The 3D edge transport code, EMC3-EIRENE, has been implemented on the ITER start-up limiter configuration, in order to analyze 3D transport properties in geometry of the toroidally discrete limiter and to investigate the power load on the limiter surface. The main results of the analysis can be summarised as follows:

1. Because of the resonant feature of field line trajectories at rational $q$ surfaces, the toroidally discrete limiters in the edge region produces a complex 3D pattern in the connection length profiles, where long and short flux tubes co-exist in the scrape-off layer (SOL). 
2. The severity of problems associated with very long flux tubes in the edge, which can bring a large amount of energy (proportional to $\sqrt{L_{C}}$ ), and cause a hot spot on the limiter, was mitigated and no significant localized power load was found. This can be justified as follows: (i) For long flux tubes, at the region of $\mathrm{s}>500 \mathrm{~m}$, where $\mathrm{s}$ is a distance from the limiter, the perpendicular energy transport time becomes shorter than the parallel energy transport time, $\tau_{E \perp}<\tau_{E / /}$, resulting in no net energy input to the flux tube. (ii) Additionally, perpendicular transport was found to be very effective to smear out the difference in // energy flux conducted by the various flux tubes, if they interact within a perpendicular transport scale, $\Delta r_{C}=\sqrt{2 \chi_{\perp} \tau_{E / /}} \sim$ a few $\mathrm{cm}$, which is usually the case in high plasma current ITER start-up configuration. These two effects significantly reduce the dependence of energy deposition, $\Gamma_{/ /} \propto \sqrt{L_{C}}$.

3. The results presented are affected by the existing uncertainties of the transport coefficients, $D_{\perp}$ and $\chi_{\perp}$. A parametric scan was carried out within a range estimated from the JET limiter discharge [8,9]). At the smaller plasma current (e.g., $\mathrm{I}_{\mathrm{p}}=2.5 \mathrm{MA}$ ), because of the larger $D_{\perp}$ and $\chi_{\perp}$, the $\lambda_{\mathrm{p}}$ becomes longer and the power is deposited at the limiter edge. Due to the low $\mathrm{P}_{\mathrm{SOL}}$ in the smaller $\mathrm{I}_{\mathrm{p}}$, however, the peak power load is still far below the engineering limit. At higher plasma currents (e.g., $\mathrm{I}_{\mathrm{p}}=6.5 \mathrm{MA}$ ), the peak power load is found to be close to the engineering limit, $8 \mathrm{MW} / \mathrm{m}^{2}$ [1,2], especially for lowest values $D_{\perp} \& \chi_{\perp}$ and highest $\mathrm{P}_{\mathrm{SOL}}$.

The results suggest that the limiter shape has to be optimized to the higher Ip, because of the higher $\mathrm{P}_{\mathrm{SOL}}$ and of the shorter $\lambda_{\mathrm{p}}$ due to the smaller $D_{\perp} \& \chi_{\perp}$.

4. The radial decay of $\Gamma_{/ /}$obtained by the $3 \mathrm{D}$ modelling was found to be not a simple exponential decay. There exists also decay in the poloidal direction, which is not taken into account in a simple model.

Comparing the results of this assessment with those obtained by modelling with a simple model [14], it was found that by neglecting the 3D geometrical effects, the simple model overestimates peak power load by $\sim 30 \%$ for corresponding input power and $\lambda_{\mathrm{p}}$.

\section{Acknowledgements}

The authors are grateful to Dr. R. Mitteau for the fruitful discussions. One of the authors, M. Kobayashi, is also grateful to Dr. Y. Shimomura, Prof. O. Motojima and Prof. N. Ohyabu, for their continuous encouragement on the accomplishments of this task.

\section{References}

[1] Cardella A, Skladnov K, Ioki K, Pacher H, Strebkov Y and Daenner W 2002 Fus. Eng. Des. 61-62 111

[2] Cardella A, Lodato A, Pacher H D, Parker R R, Ioki K, Janeschitz G, Lousteau D, Raffray R, Yamada M and Gusic C 1998 Fus. Eng. Des. 4375

[3] Pacher H D and Pacher G W "ITER Startup Limiter Power Loads", ITER Final report 2001.

[4] Feng Y, Sardei F, Kisslinger J, Grigull P, McCormick K and Reiter D 2004 Contr. Plasma Phys. 4457 
[5] Reiter D, Baelmans M and Börner P 2005 Fusion Science and Technology 47 172-186, and www.eirene.de (2004).

[6] ITER Final Report for Task Agreement N 19 TD 04 FJ (2003).

[7] Federici G, Zolotukhin O, Tanga A, Loarte A, Horton L, Portone A and Kobayashi M "Simulations of ITER start-up for limiter power load assessment" ITER_D_2229DE v1, Jan. 2006

[8] Erents S K, Tagle J A, McCracken G M, Stangeby P C and De Kock L 1988 Nucl. Fusion 28 1209

[9] Tagle J A, Erents S K, McCracken G M, Pitts R A, Stangeby P C, Lowry C and Stamp M F 1987 14th EPS Madrid 11C partII p.662

[10] Lehnen, M., et al., Nucl. Fusion 43 (2003) 168.

[11] Kim, J.W., et al., Journal of Nuclear Materials, 290-293 (2001) 644.

[12] Loarte, A., Journal of Nuclear Materials, 266-269 (1999) 1123.

[13] ITER Physics Basis Editors, Nucl. Fusion 39 (1999) 2137.

[14] Federici G, Strohmayer G, Loarte A and Kobayashi M, "Modelling of Power Handling of the Beryllium Start-up Limiters of ITER” ITER report being prepared.

[15] Stangeby P C, Pitcher C S and Elder J D 1992 Nucl. Fusion 322079

[16] Rozhansky V A et al., Nucl. Fusion 41 (2001) 387.

[17] Rozhansky V A Plasma Phys. Control. Fusion 46 (2004) A1. 